\title{
Particle-Level Visualization of Hydrodynamic and Frictional Couplings in Dense Suspensions of Spherical Colloids
}

\author{
Taiki Yanagishima $\odot,{ }^{1}$ Yanyan Liu, ${ }^{1}$ Hajime Tanaka $\odot,{ }^{2,3, *}$ and Roel P. A. Dullens $\circledast^{1, \dagger}$ \\ ${ }^{1}$ Department of Chemistry, Physical and Theoretical Chemistry Laboratory, \\ University of Oxford, South Parks Road, Oxford OX1 3QZ, United Kingdom \\ ${ }^{2}$ Department of Fundamental Engineering, Institute of Industrial Science, \\ The University of Tokyo, Komaba 4-6-1, Meguro-ku, Tokyo 153-8505, Japan \\ ${ }^{3}$ Research Center for Advanced Science and Technology, University of Tokyo, \\ 4-6-1 Komaba, Meguro-ku, Tokyo 153-8505, Japan
}

(Received 1 September 2020; revised 15 March 2021; accepted 15 April 2021; published 14 June 2021)

\begin{abstract}
The rotational Brownian motion of colloidal spheres in dense suspensions reflects local hydrodynamics and contact forces, which are both key to nonlinear rheological phenomena such as shear-thickening and jamming, and transport in crowded environments, including intracellular migration and blood flow. To fully elucidate the role of rotational dynamics experimentally, it is crucial to measure the translational and rotational motion of all spheres simultaneously. Here, we directly access hydrodynamic and frictional coupling in colloidal suspensions up to arbitrarily high volume fractions using compositionally uniform colloidal spheres with an off-center, fully embedded core. We reveal interparticle hydrodynamic rotationrotation coupling in charged colloidal crystals. We also find that higher local crystallinity in denser hardsphere crystalline sediments enhances rotational diffusivity and that nearly arrested particles exhibit a stick-slip rotational motion due to frictional coupling. Our findings shed new light on the largely unexplored, local rotational dynamics of spherical particles in dense particulate materials.
\end{abstract}

DOI: $10.1103 /$ PhysRevX.11.021056

Subject Areas: Condensed Matter Physics, Soft Matter

\section{INTRODUCTION}

The dynamics of colloidal particles is key to connecting the equilibrium phase behavior of particulate suspensions to their atomic analogs [1,2]. The vast majority of studies of condensed matter phenomena only focus on translational degrees of freedom; examples include crystallization [3-5], melting [6,7], gelation [8,9], and the glass transition [10] (see Ref. [11] for a review). However, this is only half the picture. As noted from the outset by Perrin [12], colloidal particles also feature rotational Brownian motion, which includes spherical particles that do not have an easily visualized orientation.

For a suspension of spherical particles, rotational Brownian motion is governed by two physical effects, hydrodynamics and contact forces, including friction. Nonlocal hydrodynamic interactions play an important role in colloidal gelation [9,13-15], the rheology of complex

\footnotetext{
tanaka@iis.u-tokyo.ac.jp

roel.dullens@chem.ox.ac.uk
}

Published by the American Physical Society under the terms of the Creative Commons Attribution 4.0 International license. Further distribution of this work must maintain attribution to the author(s) and the published article's title, journal citation, and DOI. fluids [16,17], and biological systems [18-20]. Contact forces such as adhesion and friction also play a significant role in the behavior of colloidal systems. In the selfassembly of DNA-coated colloids, irreversible adhesion can prevent the formation of target structures, while a system that allows colloidal "rolling" allows equilibration to the intended state [21]. The role of interparticle friction in rheological phenomena such as shear thickening (reviewed in Ref. [22]) is also of particular interest due to its industrial relevance. Extensive experimental [23-28] and theoretical [29-32] work in both athermal and thermal regimes has led to a significant debate on the relative roles of contact interactions and hydrodynamics in shear thickening. Notably, microscopic access to a link between structure and rheology has become possible by confocal rheology $[23,25,33]$. Nevertheless, direct access to the rotational degrees of freedom of spherical particles, which allows access to hydrodynamic and mechanical interactions at the particle level, has been challenging to achieve.

Although orientational dynamics have been studied extensively for anisotropic particles [34-36], there are exceedingly few studies of rotational fluctuations in dense suspensions of spheres because of the lack of a colloidal model system that allows both the position and orientation of all the spheres in a field of view to be imaged up to arbitrarily high volume fractions. Studies using light 
scattering have given us valuable insights into rotational diffusion in suspensions [37-39], but they have been unable to correlate dynamics with local structure to reflect structural heterogeneity, a key strength of mesoscopic colloidal models.

Recently, there has been a clear escalation of efforts to create a viable system. Efforts to directly image rotational dynamics include nematic liquid crystal droplets with a frozen director [40], Janus (MOON) particles [41], anisotropic fluorescence profiles using photobleaching $[42,43]$, and rough colloidosomes with a subpopulation of fluorescent surface probes [44], but none of these allows confocal microscopy studies at arbitrarily high volume fractions. A recent work encapsulated a silica core [45] in a 3-trimethoxysilyl propyl methacrylate (TPM) shell to make a core-shell particle, again leaving a scattering interface that makes confocal microscopy in dense suspensions unfeasible. Anisotropic polymethyl methacrylate clusters in a spherical polymethyl methacrylate shell were proposed to rectify this [46], but producing monodisperse batches in bulk is not possible. As of yet, no system allows full threedimensional (3D) characterization of rotational fluctuations in monodisperse spheres using confocal laser scanning microscopy (CLSM) in arbitrarily dense systems.

Here, we directly visualize hydrodynamic and contact forces in dense colloidal suspensions by applying monodisperse colloidal spheres with uniform composition and a nonuniform fluorescence profile. The particles can be density and index matched in a solvent mixture, allowing a single 3D confocal microscopy snapshot to reveal the coordinates and orientations of all observed particles up to arbitrarily high concentrations. Using these probes, we study hydrodynamic coupling in charged colloidal crystals, finding that the rotation of adjacent spheres exhibits transient coupling. We also explore higher concentrations in a solvent with negligible particle charging and strong electrostatic screening; we study a dense, partially crystalline sediment, finding, for the first time, a positive correlation between rotational diffusivity and local crystallinity. Finally, we observe a stick-slip dynamics, indicative of the emergence of local contact friction.

\section{COLLOIDAL SPHERES FOR TRACKING 3D ROTATIONAL DYNAMICS}

To facilitate the elucidation of rotational dynamics up to arbitrarily high densities, we start by producing monodisperse, compositionally homogeneous colloidal spheres with a core-shell structure, where the core is located just below the surface; the core and shell are both TPM but labeled with different dyes (see Appendix A). Given the lack of a name for such spheres, we call these particles "offcenter core under laser illumination" (OCULI) particles, and hereafter, we refer to the core as the "eye" and to the whole particle as the "body." Next, we coat these particles with a nonfluorescent layer to make core-shell OCULI, particles compatible with individual particle tracking all the way up to close packing: it is at these high volume fractions that hydrodynamic and contact mechanics begin to play a role. Importantly, TPM particles can also be transferred into a density- and index-matching solvent mixture [47,48], allowing imaging deep inside dense suspensions.

\section{A. Synthesis of OCULI and core-shell OCULI particles}

A schematic of core-shell OCULI synthesis is given in Fig. 1(a) together with fluorescence (FL) and bright-field (BF) microscopy images in (b) and scanning electron microscopy (SEM) images in (c). Details are given in Secs. VIB and VIC. Monodisperse, fluorescent TPM particles [47] are suspended in a basic solution [Fig. 1(b1)]. Prehydrolyzed TPM (hTPM) is added, allowing TPM to condense onto the particles until it engulfs them. Interface pinning [49] and a low wetting angle between hTPM and the core ensures that the core is localized just inside the surface, as shown in Fig. 1(b2). The body is labeled using a spectrally distinct dye and cross-linked. The result is an offcenter core-shell particle that is compositionally isotropic except for a trace amount of dye, which is critical for realizing a spherically symmetric and isotropic particle with no gravitational bias. SEM images [Figs. 1(c1)and 1(c2)] show that both the core and the OCULI particles are spherical and smooth. A two-channel 3D-CLSM image in an index-matching mixture of trichloroethylene (TCE) and tetralin is also shown in Fig. 1(d). A video is provided as Supplemental Material [78]. For this study, we choose BDP-FL for the eye and Cyanine3 for the body. Recently, we learned that these OCULI particles were developed independently [50] in a different context, again highlighting the urgency with which such probes are sought after.

Fully fluorescent particles allow particle localization when interparticle contact is rare. At higher volume fractions, when contact and hydrodynamic interactions are expected to emerge, localization becomes prohibitively difficult using 3D-CLSM due to overlapping point-spread functions [51]. In the same spirit as symmetric core-shell particles [52,53], we add an extra nonfluorescent layer of TPM to the OCULI particles following Ref. [47], as illustrated in the latter half of Fig. 1(a). OCULI particles are exposed to Pluronic F108 before cross-linking; adding hTPM now nucleates small surface lobes to make raspberrylike particles [see Figs. 1(b3) and 1(c3)]. These particles are cross-linked and exposed to more hTPM, filling the gaps and making the particles spherical again. Figure 1(b4) shows a bright-field image with added fluorescence excitation of the OCULI body inside. A confocal image is also given in Fig. 1(e), suspended in an index-matching solvent mixture with a trace amount of BDP-FL dye, like the eye. A dark, nonfluorescent layer is clearly visible at the surface, while the central part of the particle maintains the unique fluorescence profile of the OCULI particles. SEM again confirms that the core-shell OCULI particle is also spherical and 
(a)
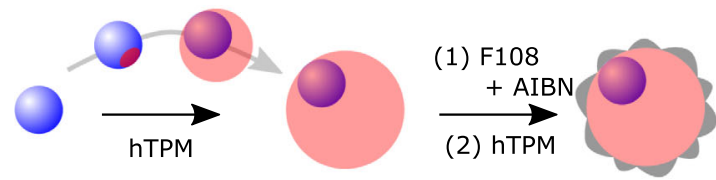

$\underset{\text { (2) } \mathrm{hTPM}}{\stackrel{\text { (1) AIBN }}{\longrightarrow}}$

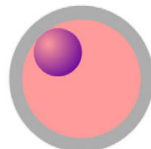

OCULI
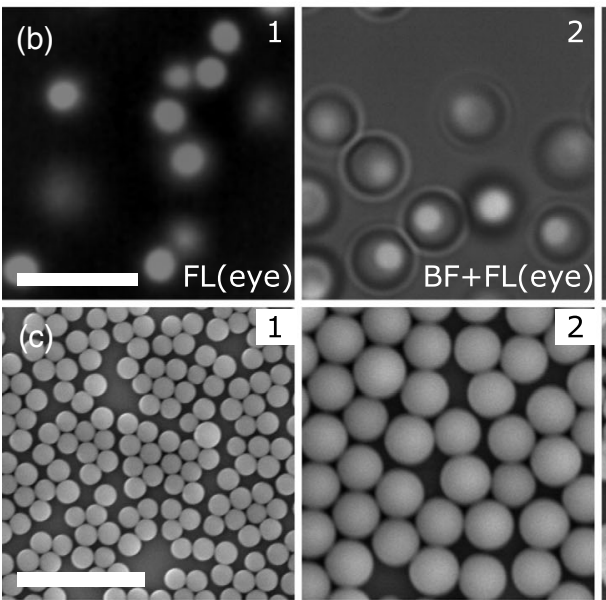
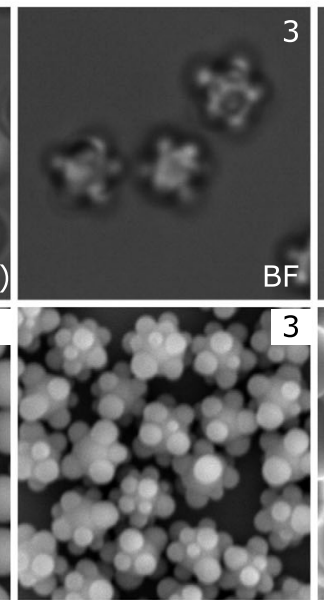

Core-shell OCULI

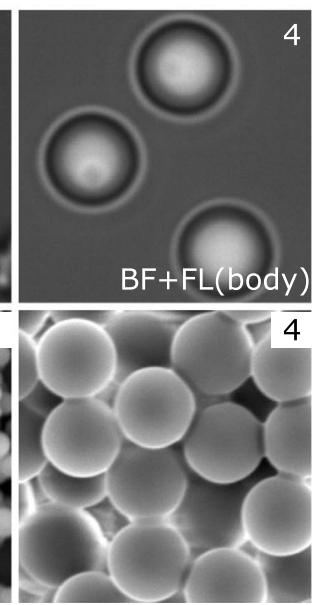

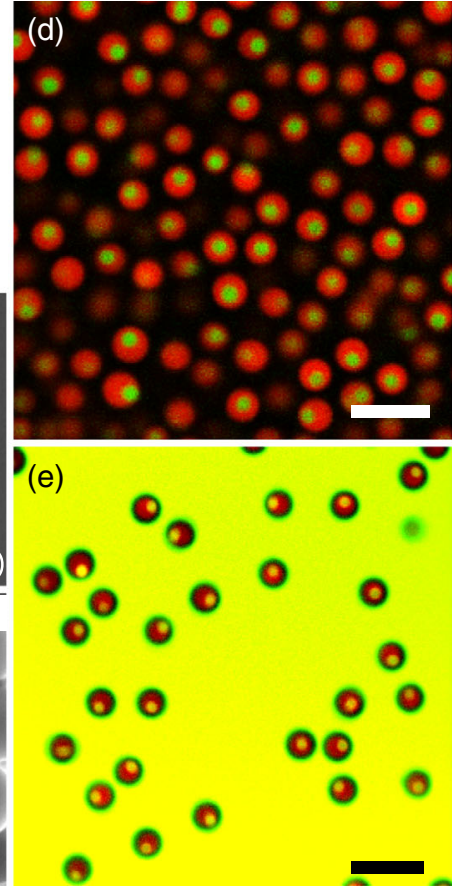

FIG. 1. Synthesis of OCULI and core-shell OCULI particles. (a) Schematic of how monodisperse TPM particles are embedded in prehydrolyzed TPM (hTPM) to form off-centered OCULI particles. Further coating by hTPM in a two-step procedure via intermediate raspberry particles results in "core-shell OCULI" particles. (b1) Fluorescence image of TPM "eye" particles. (b2) OCULI particles, imaged in bright field with fluorescence excitation of the eye. (b3) Raspberry OCULI particles, imaged in bright field. (b4) Core-shell OCULI particles, imaged in bright field with fluorescence excitation of the OCULI body. (c) SEM pictures of particles in (b). (d) Twochannel confocal microscopy image of OCULI particles. (e) Two-channel confocal microscopy image of core-shell OCULI particles. The solvent is also dyed with a trace amount of BDP-FL. All scale bars are $5 \mu \mathrm{m}$.

smooth [see Fig. 1(c4)]. A detailed protocol is provided in Appendix A.

\section{B. Tracking the rotational motion of individual particles}

To study the rotational dynamics of each particle using 3D-CLSM, we apply conventional particle-tracking methods to obtain eye and body positions separately. We stress that this is possible even at higher concentrations due to the separation between fluorescence profiles afforded by the core-shell OCULI. An example is given in Fig. 2; a two-channel slice of a 3D-CLSM stack is shown in (a). Eyes were tracked using a variant on Ref. [54]: the eye signal (c1) is smoothed before a Gaussian fit around the maximum gives a position with subpixel accuracy (c2). The body is located by taking the body signal (b1), bandpassing it (b2), and binarizing (b3) before resmoothing using a Gaussian kernel (b4) to reduce any bias introduced by the eye. Subpixel accuracy is achieved with a quadratic fit around the maximum. Eyes are associated with bodies by a simple distance threshold, as shown in Fig. 2(d). The vectors joining them are normalized to give a unit orientation vector $\boldsymbol{u}(t)$ associated with each particle. Standard methods [54] are used to associate body positions into trajectories over time. Particles with multiple eyes $(<0.5 \%$ of total) are removed from the analysis.

\section{ROTATIONAL DYNAMICS OF ALL SPHERES IN COLLOIDAL MATERIALS}

Rotational Brownian motion at high densities is chiefly governed by nonlocal hydrodynamic and local contact interactions. To quantitatively address these phenomena and elucidate rotational correlations, it is a key prerequisite to have access to both the position and orientation of all the spheres in 3D. We demonstrate this for the first time in dense materials using OCULI and core-shell OCULI particles. In particular, we elucidate hydrodynamic coupling in charged colloidal crystals and the relation between rotational diffusivity, crystallinity, and contact mechanics at the single-particle level in dense sediments.

\section{A. Hydrodynamic coupling in charged colloidal crystals}

When charged particles are not in contact, their rotational motion can couple through hydrodynamic interactions. Colloidal particles in external fields are also known to assemble via hydrodynamically mediated mechanisms; e.g., polystyrene spheres in an alternating field crystallize via collective rotational motion [55]. Rotating colloids 


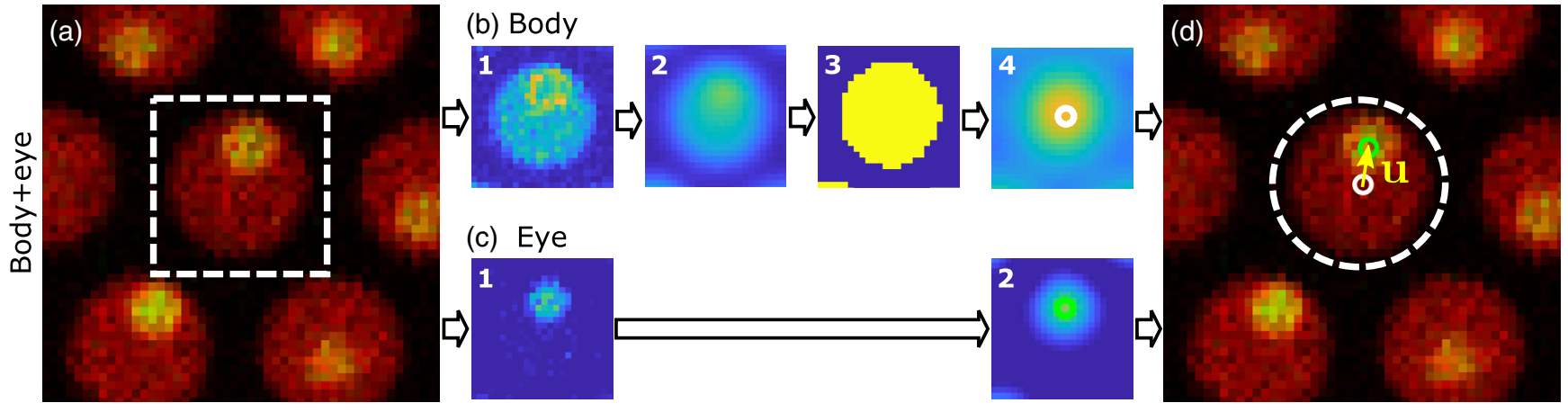

FIG. 2. Orientation vector location from confocal microscopy. (a) Composite image of a single slice from a two-channel 3D-CLSM stack. (b) Particle location for the particle body. The raw data for the corresponding channel (1) are band passed (2), binarized (3), and then resmoothed (4) before a particle location algorithm is applied [point in (4)]. (c) Particle location for the particle eye. The raw data for the corresponding channel (1) are band passed (2) before a particle location algorithm is applied [point in (2)]. (d) Body and eye positions are associated via a simple distance threshold (dashed circle) and joined to give a particle-specific orientation vector, $\boldsymbol{u}$.

are even known to possess phases created purely by hydrodynamic interactions [56,57]. To understand how rotational motion, both passive and driven, underpins assembly and transport, it is crucial to have a complete visualization of both particle orientations and positions.

To demonstrate the utility of such a measurement, we study 3D rotational Brownian motion in a low-density crystal of charged OCULI particles in a large, 3D region of interest. We specifically choose a solvent mixture and stabilizer concentration that leads to particle charging and moderate electrostatic screening, a 1:1 v/v mixture of TCE and tetralin with $0.5 \mathrm{wt} \%$ OLOA 1200 stabilizer. From previous work [48], we estimate that the system has a Debye length of $\lambda_{D} \approx 170 \mathrm{~nm}$. The particles are $1.90 \mu \mathrm{m}$ in diameter with a polydispersity of $3.6 \%$ (sized with SEM); thus, as a ratio of particle size, $\sigma \lambda_{D}^{-1} \approx 10$. Despite the crowding, the particles are sufficiently separated by electrostatic repulsion to ensure that any rotational correlation is ascribed to hydrodynamic interactions. The absence of long-time translational diffusion also allows for an effective sampling of rotational motion of pairs of particles at certain interparticle separations. A confocal microscopy image is given in Fig. 3(a); the (1 111 ) plane of a face-centered cubic crystal is parallel to the base of the sample cell [5]. Though the refractive index of the particles is matched to the solvent to allow 3D-CLSM imaging, the mass density is mismatched, leading to slow sedimentation and subsequent crystallization. The volume fraction $\phi$ in the crystal is about 0.18 , which is estimated using the SEM particle size and a 3D Voronoi tessellation.

We first consider single-particle rotational diffusion, calculating the autocorrelation of the unit orientation vectors $\boldsymbol{u}_{i}(t)$ of each OCULI particle $i$. When a spherical particle undergoes diffusive rotational motion, the autocorrelation function $C(t)$ is expected to decay exponentially,

$$
C(t)=\langle\boldsymbol{u}(t) \cdot \boldsymbol{u}(0)\rangle=e^{-t / \tau_{r}},
$$

where $\tau_{r}=1 / 2 D_{r}$, with $D_{r}$ the rotational diffusion constant. A derivation reproduced from Ref. [2] is given in Appendix B. At infinite dilution, $D_{r}$ is given by $D_{r}=$ $k_{\mathrm{B}} T / \pi \eta \sigma^{3}$ for nonslip boundary conditions, where $k_{\mathrm{B}} T$ is the thermal energy, $\eta$ is the effective viscosity of the surrounding medium, and $\sigma$ is the diameter of the particle. Here, $C(t)$ averaged over all particles is shown in Fig. 3(b), and it is well described by the exponential decay of Eq. (1). This shows that the rotational motion of the OCULI spheres, despite their proximity to other particles in the crystal, is purely diffusive at the single-particle level, consistent with previous light-scattering experiments [37]. Given knowledge of individual $C(t)$ for all the spheres, we also look at the distribution of relaxation times [Fig. 3(b), inset]. While the relaxation times from individual particles converge around the average at $\tau_{r}=5.8 \pm 0.1 \mathrm{~s}$, the distribution exhibits a tail towards longer relaxation times. The average relaxation time is also longer than what is expected at this volume fraction [37], $\tau_{r}(\phi=0.18)=3.47 \mathrm{~s}$ (see Appendix E). We attribute this to the gravitational compression of the crystal: the crystal planes are compressed along the vertical direction, making interparticle separations across adjacent $\left(\begin{array}{lll}1 & 1 & 1\end{array}\right)$ crystal planes smaller than those within the same plane. This is also apparent from the distribution of particle separations $p(r)$ in Fig. 3(c), where a splitting is seen in the first peak due to interlayer and intralayer particle separations. This compaction should enhance the coupling between the particle-size polydispersity and the rotational relaxation. The hydrodynamic friction is also significantly stronger for particle pairs of larger size; this may be the origin of the long $\tau_{r}$ tail in $p\left(\tau_{r}\right)$. Note that hydrodynamic drag strongly increases with a decrease in the interparticle distance $r$. 
(a)

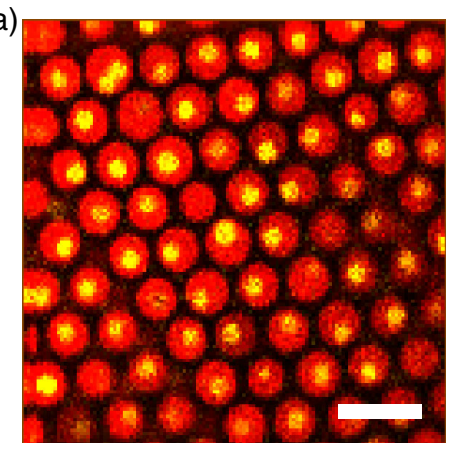

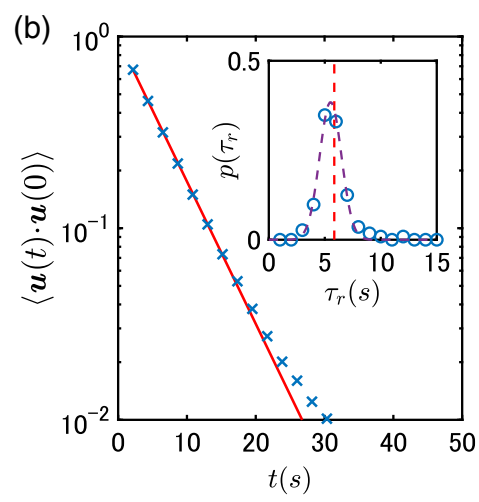

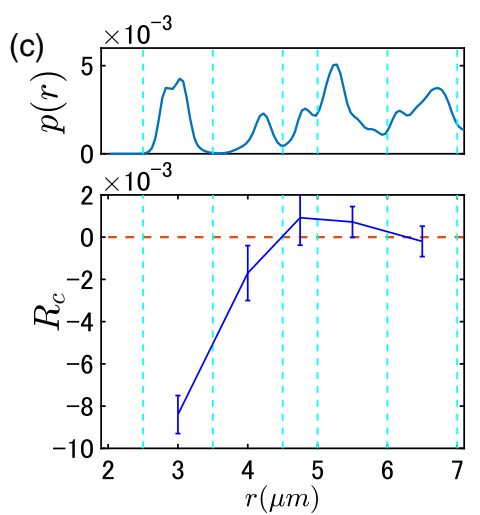

FIG. 3. Rotational motion in a charged colloidal crystal of OCULI particles. (a) Two-channel confocal microscopy image of OCULI particles. The scale bar is $5 \mu \mathrm{m}$. (b) Average autocorrelation function of unit orientation vectors over time, and an exponential fit (red solid line). Inset: distribution of relaxation times for single particles in a field of view (symbols) and a Gaussian fit (purple dashed line). The red vertical dashed line shows the average relaxation time found from the exponential fit to the average autocorrelation function. (c, upper) Probability distribution $p(r)$ of interparticle distances in the charged crystal. (c, lower) Rotation-rotation coupling constant $R_{c}$ [Eq. (2)] for different coordination shells around a central particle (separated by vertical light blue dashed lines) for $\tau_{r} \approx 2 \mathrm{~s}$. Error bars correspond to the standard error in the mean value of $R_{c}$ for particles in each $r$ bin.

With access to the orientations of neighboring spheres, we now directly quantify hydrodynamic rotation-rotation coupling between spheres in different coordination shells as identified in $p(r)$ [see Fig. 3(c)]. We estimate the angular velocity vector $\omega_{i}$ of particle $i$ rotating from $\boldsymbol{u}_{i}(t)$ to $\boldsymbol{u}_{i}(t+\tau)$ over a time $\tau$ as $\boldsymbol{\omega}_{i}=\boldsymbol{u}_{i}(t+\tau) \otimes$ $\boldsymbol{u}_{i}(t)$. We thus define a rotation-rotation coupling constant $R_{c}\left(r_{i j}=r, \tau\right)$, given by (see Appendix D)

$$
R_{c}(r, \tau)=\frac{\omega_{i} \cdot \omega_{j}}{\left\langle\omega_{i}^{2}\right\rangle^{1 / 2}\left\langle\omega_{j}^{2}\right\rangle^{1 / 2}}
$$

Note that $R_{c}$ for pairs of particles will be closer to 1 when they rotate in the same direction and -1 when they rotate in opposite directions. Here, $R_{c}$ for pairs of particles in the first four coordination shells is shown in Fig. 3(c) for $\tau=2.17 \mathrm{~s}$, the time between adjacent frames. We find a weak negative coupling between particles in the first coordination shell, indicating that adjacent particles are more likely to rotate in opposite directions, like meshed gears. Since the first coordination shell is at a distance of $r=3.0 \mu \mathrm{m} \approx 1.6 \sigma$, the coupling is mediated by hydrodynamic interactions. Indeed, earlier theoretical work [40] showed that antisymmetric rotation-rotation coupling between Brownian particles due to hydrodynamic effects is non-negligible for this distance. We note that the symmetry of the nearest-neighbor particle arrangement in the crystal is incompatible with persistent rotational coupling. Yet, despite the frustration, the rotational coupling is persistent enough to be detectable using these particles, though it is clearly spatiotemporally heterogeneous.

\section{ROTATIONAL DIFFUSIVITY AND LOCAL CRYSTALLINITY IN DENSE, PARTIALLY CRYSTALLIZED, HARD-SPHERE SUSPENSIONS}

\section{A. Spatial heterogeneity of rotational diffusivity}

Pioneering work on rotational diffusion in dense suspensions [37,58] has measured and simulated how rotational diffusivity varies with volume fraction in density-matched suspensions, with accurate predictions for $D_{r}$ using a virial expansion. While these works highlight the ultrasensitivity of $D_{r}$ to short-range interactions and the pair distribution function, the effect of local structure and gravity on local rotational diffusivity remains unaddressed. Access to complete knowledge of $D_{r}$ of all individual spheres puts us in a unique position to address these ideas in detail. Thus, we form a dense, partially crystalline sediment using core-shell OCULI spheres, as shown in Fig. 4(a). The nonfluorescent surface layer ensures a clear separation of fluorescence signals, facilitating accurate particle tracking (see Sec. II B). The particles have a total diameter of $\sigma=2.81 \mu \mathrm{m}$ (SEM) with a polydispersity of $2.9 \%$ and are suspended in a 3:1 v/v mixture of TCE and tetralin. In contrast to our study of charged crystals, we choose a species and concentration of stabilizer that produces hard-sphere-like interactions, $5 \mathrm{wt} \%$ of OLOA 11000. Experimental characterization using optical trapping in this specific solvent mixture is also shown in Appendix F, which explicitly demonstrates the lack of any significant charging or long-ranged repulsion. Given negligible electrostatic interactions at 100 -nm separation, we may estimate an upper limit for the Debye length such that $\lambda_{D} \ll 100 \mathrm{~nm}$, which would imply $\sigma \lambda_{D}^{-1} \gg 30$. Using bond orientational order parameters, we find that $34.3 \%$ of the sample is crystalline, corresponding to an effective 
(a)

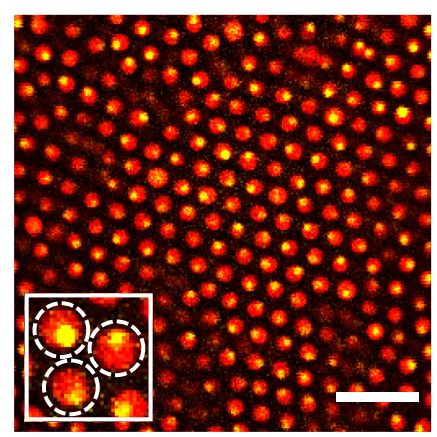

(d)
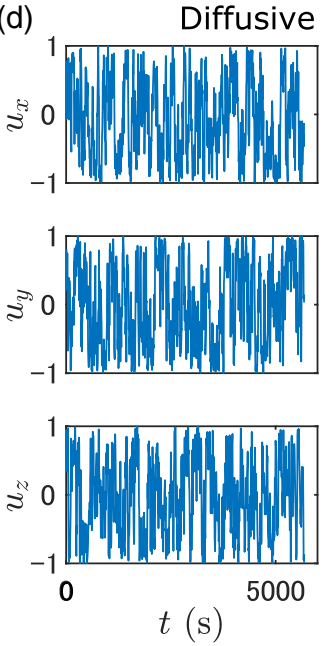
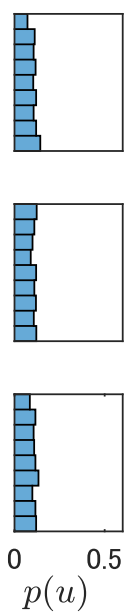

(b)
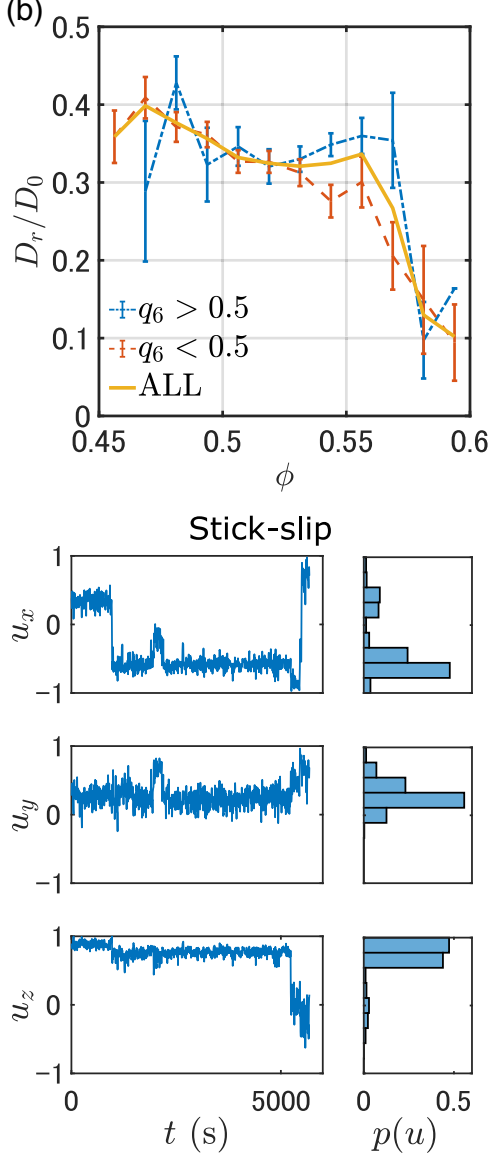
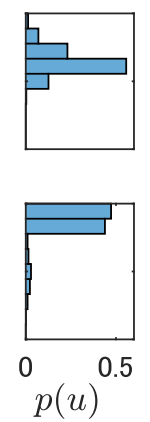

(c)

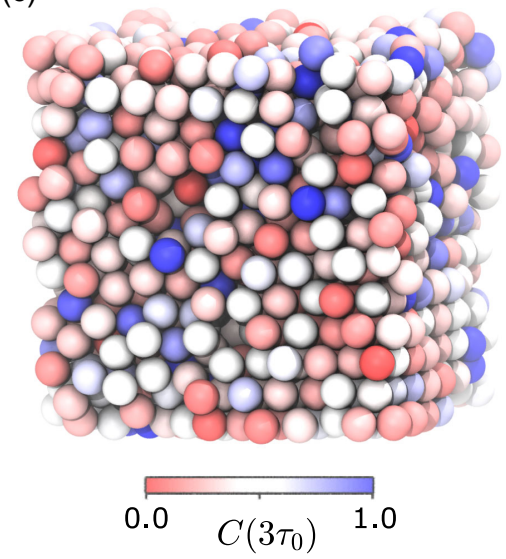

(e)

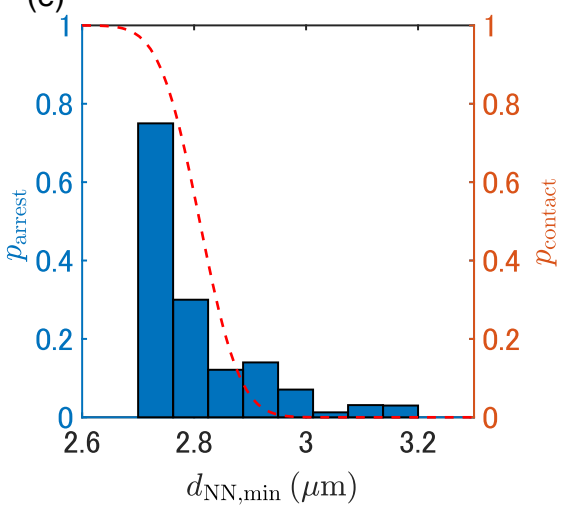

FIG. 4. Rotational motion in a partially crystalline sediment of core-shell OCULI particles. (a) Two-channel confocal image of coreshell OCULI particles. The scale bar is $10 \mu \mathrm{m}$. Inset: zoomed-in portion with effective hard-sphere size indicated. (b) Rotational diffusivity of particles as a function of local volume fraction $\phi$. Statistics are taken over three populations: crystalline particles $\left(q_{6}>0.5\right)$, amorphous particles $\left(q_{6}<0.5\right)$, and all particles. (c) Rendering of the particles in the region of interest, color coded by $C\left(3 \tau_{0}\right)$ values: Red is for low, and blue is for high, scaled over the entire range $0<C<1$. (d) Unit orientation vector components $u_{x}, u_{y}$, $u_{z}$ as a function of time for particles undergoing diffusive $\left[C\left(3 \tau_{0}\right) \approx 0.06\right.$, left $]$ and stick-slip $\left[C\left(3 \tau_{0}\right)>0.9\right.$, right $]$ rotational motion, and their histograms. (e) Probability of a particle being rotationally arrested, $p_{\text {arrest }}=p\left(C\left(3 \tau_{0}\right)>0.9\right)$, and the probability of two particles being in contact at a particle separation, $p_{\text {contact }}$.

hard-sphere volume fraction of about $51 \%$ (see Appendix G) and an effective hard-sphere size of $3.00 \mu \mathrm{m}$ (consistent with the SEM diameter swelled slightly in the solvent). This diameter is indicated as an inset in Fig. 4(a) for clarity; it should be stressed that this system is highly screened and not significantly charged.

First, we consider the autocorrelation function $C(t)$ of $\boldsymbol{u}(t)$ for each particle and measure the rotational diffusivity with an exponential fit via Eq. (1), using $\tau_{r}=1 / 2 D_{r}$. Hence, we obtain the rotational diffusivity relative to that at infinite dilution $D_{r} / D_{0}$ by calculating $D_{r} / D_{0}=\tau_{0} / \tau_{r}$. Here, $\tau_{0}$ is the rotational relaxation time at infinite dilution, calculated using the hydrodynamic diameter (see Appendix C). This quantity is plotted as a function of the effective local volume fraction $\phi$ in Fig. 4(b). Generally, rotational diffusion slows down upon increasing the volume fraction, consistent with earlier light scattering and tracer-based experiments [37-39,46].
However, there are some key differences with previous work. First, we find that $D_{r} / D_{0}$ is smaller than in previous findings $[37,58]$. Furthermore, we see that at higher local volume fractions, $0.52<\phi<0.56$, there is in fact a counterintuitive and surprising plateauing of $D_{r} / D_{0}$ with increasing $\phi$ [see solid curve in Fig. 4(b)]. Previous work found a small leveling-off of translational and rotational diffusivity through the freezing point with increasing $\phi$ [37]; for translational diffusivity, this was attributed to the increase in free volume upon crystallization. Note that previous works described the dependence of the average $D_{r}$ on an average volume fraction; we now have access to the dependence of $D_{r}$ of individual particles on their local volume fraction $\phi$. Figure 4 (b) shows $D_{r} / D_{0}$ as a function of $\phi$ for crystalline particles (blue-dashed curve, $q_{6}>0.5$ ) and amorphous particles (orange dashed curve, $q_{6}<0.5$ ). Strikingly, the crystalline particles exhibit a distinct transient increase in $D_{r}$, while $D_{r}$ for the amorphous particles 
shows a monotonic decrease with $\phi$. Importantly, the two lines clearly lie on either side of the all-particle average. The trend is robust against a whole range of $q_{6}$ thresholds taken between the peaks corresponding to the crystal and liquid phases in Fig. 7(d). To the best of our knowledge, this is the first experimental verification of local crystallinity enhancing rotational diffusion.

The fact that an environment with higher order provides less hydrodynamic friction may be rationalized by considering that it is the shortest interparticle separation that mainly contributes to interparticle hydrodynamic friction. This may be demonstrated directly by considering the shortest interparticle distances for the same particle population as those shown in Fig. 4, simply finding the distance to the closest nearest-neighbor particle (averaged over 20 time steps). This method is shown in Appendix $\mathrm{H}$. We can see a clear difference between the distances for crystalline and noncrystalline particles, in line with Fig. 4(b), where the closest nearest neighbor tends to be closer for noncrystalline particles.

We also directly visualize the rotational heterogeneity in Fig. 4(c) by coloring the particles according to their value of $C(t)$ at $t=3 \tau_{0}$. We choose $t=3 \tau_{0}$ to allow for a clear differentiation between slow and fast rotational dynamics: Red is $C\left(3 \tau_{0}\right)=0.0$ (fast diffusion, completely decorrelated), and blue is $C\left(3 \tau_{0}\right)=1.0$ (completely arrested). While the heterogeneity is obvious, we also note that there is a subpopulation of particles that are nearly entirely arrested, which will be discussed later.

It remains to address why the rotational diffusivity in our partially crystalline sediment is relatively slow compared to previous studies $[37,58]$ [Fig. 4(b)] and remarkably heterogeneous [Fig. 4(c)]. First, we note that gravity in our system induces significantly more interaction between particles in different layers perpendicular to the gravitational direction, particularly in the absence of significant long-range repulsion. The gravitational Peclet number for this system is 0.45 , which is vastly different from density-matched scattering experiments. It is still small enough $(<1)$ to observe some diffusive effects but large enough to ensure persistent particle proximity and a significant reduction of interparticle spacings, leading to a reduction in rotational diffusivity. This effect is more pronounced when the particle arrangement is amorphous, as demonstrated. Second, in the absence of significant charging, particles may even be driven to come into contact with each other, giving rise to a contact interaction, which is not accounted for in existing theories. Not only would such an effect significantly reduce diffusivity, but it would also amplify the difference between crystalline and amorphous particles, which is exactly what we observe.

\section{B. Emergence of contact interactions}

The effect of contact forces may be directly probed using our OCULI particles, which is pivotal to the potential impact these particles may have in other fields. Friction, for example, is a key topic for interfacial phenomena at the atomic level [59,60] and in granular materials [61-64], where it may underpin nonlinear rheological phenomena such as discontinuous shear thickening [24,26,31]. Some claim that the interparticle frictional profile has a profound effect on suspension behavior [27]. This claim underlines another key utility of our OCULI system, i.e., how the characterization of rotational dynamics with single-particle resolution may reveal a spatiotemporal map of frictional forces acting throughout a colloidal packing.

To elucidate the role of contact forces in our partially crystalline sediment, we focus on the subpopulation of particles for which $C\left(3 \tau_{0}\right)>0.9$, i.e., are nearly arrested [see dark blue particles in Fig. 4(c)]. These nearly arrested particles fluctuate in a qualitatively different manner from those with lower $C\left(3 \tau_{0}\right)$. Comparing with how the vector $\boldsymbol{u}(t)=\left(u_{x}, u_{y}, u_{z}\right)$ fluctuates for a particle in the same sample undergoing diffusive motion $\left[C\left(3 \tau_{0}\right) \approx 0.06\right.$; see Fig. 4(d), left], we see that these nearly arrested particles exhibit an intermittent rotational motion [see Fig. 4(d), right], with clear peaks in the histogram of $u_{x}, u_{y}$, and $u_{z}$. This case applies to approximately $70 \%$ of the particles with $C\left(3 \tau_{0}\right)>0.9$. Interestingly, this motion is uncorrelated with the translational and rotational motion of its neighbors (see Appendix I). Instead, it is closely correlated with the distance to the closest nearest neighbor $d_{\mathrm{NN} \text {,min }}$, as shown in Fig. 4(e). The probability of a particle being rotationally arrested $p_{\text {arrest }}\left[C\left(3 \tau_{0}\right)>0.9\right]$ increases with shorter $d_{\mathrm{NN}, \min }$, coinciding with the rise in interparticle contact probability $p_{\text {contact }}$, estimated from the Gaussian distribution of particle sizes.

This result constitutes strong evidence that this peculiar motion arises from contact friction, where the required normal stress is provided by the weight of the particles above it, and that this intermittent dynamics is stick-slip motion. This is an essential frictional effect observed on a wide range of length scales $[26,65,66]$. Note that this effect is a very local one; one or two larger neighbors or the particle itself being larger might provide enough contact to arrest a neighbor. To the best of our knowledge, this work represents the first time stick-slip motion has been observed from the rotational Brownian motion of individual colloidal particles.

The significant number of particles undergoing stickslip motion as opposed to complete arrest is partially explained by the gravitational Peclet number for this system, which is 0.45 . The system is thus neither purely colloidal nor granular in scope; the gravitational force at play is certainly strong enough to arrest large-scale rearrangements of particles but not strong enough to completely arrest diffusive fluctuations.

It is important to note that we do not expect these particles to be perfectly smooth. The addition of an OLOA 11000 stabilizer here not only provides a screening agent 
for charge but also provides a steric repulsion by adsorbing onto the particles to form a polymer brush layer. The coverage is not expected to be dense in a random adsorptive process [67]. Atomic force microscopy and rheology measurements for OLOA 11000 adsorbed onto carbon black particles in decahydronaphthalene [68] show that the brush thickness is around $4 \mathrm{~nm}$. Given that the particles appear smooth under scanning electron microscopy with a resolution of approximately $10 \mathrm{~nm}$, we may estimate that any roughness on the bare surface is less than $10 \mathrm{~nm}$. Thus, we may conclude that the core-shell OCULI particles in this experiment have a roughness of 4-10 nm, which means that particles do not necessarily need to reach surfacesurface contact to experience a "contact" interaction.

Given the sensitivity to contact forces, it is also important that means are available to modify the surface. These particles are composed of 3-trimethoxysilyl propyl methacrylate, a common bridging component between organic and inorganic materials. Thus, any of the wealth of conjugation techniques that use silane or methacrylate chemistry may be used to modify the charge, apply a polymer brush, or change the wettability of the surface [69]. The surface roughness may also be modified by growing lobes, like we have done here in the intermediate step of the core-shell OCULI particle synthesis. This process is described in more depth in Ref. [47].

\section{CONCLUSIONS}

We have directly visualized hydrodynamic and contact interactions in dense suspensions of newly developed colloidal spheres, which enable simultaneous measurement of both the translational and rotational motion of all individual particles up to arbitrarily high volume fractions. In particular, we have measured transient rotationrotation coupling in charged colloidal crystals and also found that local crystallinity can lead to an increase in rotational diffusivity in a denser, partially crystalline sediment. Finally, we have observed the onset of stickslip rotational motion, indicating the emergence of contact friction in dense particulate materials. Importantly, our results also show that these two types of friction of hydrodynamic (fluid) and mechanical (solid) origin can be distinguished by probing the "continuity" of the rotational Brownian motion. Thus, our method allows effective detection of solidlike contact friction at the single-particle level, induced by either mechanical forces or interparticle attraction.

In order to obtain a deeper understanding of selfassembly mechanisms in flowing, driven, and nonequilibrium colloidal systems, the ability to directly image variations in the rotational dynamics of individual particles is a significant development for any particulate system where hydrodynamics, mechanical rigidity, and force chain networks play a role. Spatial mapping of rotational diffusivity may thus provide a unique strategy to visualize the incidence of jamming and allow for accurate characterization of how the statistics of local force chain networks change on approaching the jamming point. However, its greatest potential strength is to change how we scrutinize nonlinear rheological phenomena in dense slurries and pastes. Computational work clearly shows that contact friction has an important role to play in discontinuous shear thickening [27,70]; strategies are being developed to tune particle roughness to indirectly address the phenomenology [71]. The OCULI system now offers a more direct route, mapping microscopic frictional response to bulk behavior, an approach that might be applied to any number of complex rheological phenomena at the interface between colloidal and granular matter.

\section{MATERIALS AND METHODS}

\section{A. Synthesis}

Reagents, preparatory syntheses of core particles, dyes, and prehydrolyzed TPM, as well as a detailed description of the synthesis of both systems described, are given in Appendix A.

\section{B. Microscopy}

Bright-field microscopy was carried out on an IX71 frame with a 100x PlanApo oil immersion objective (Olympus). Images were taken with a XIMEA USB3.0 camera MQ042MG-CM (XIMEA). Fluorescence excitation was achieved with a CoolLED Pe-300 white light source (CoolLED).

Three-dimensional confocal laser-scanning microscopy (CLSM) was carried out using the same frame with a 60x PlanApo oil immersion objective (Olympus) and a confocal scan head with a multichannel laser source (Thorlabs). We used 532-nm and 488-nm lasers for two-channel excitation, separated with a standard FITC/Rhodamine filter cube. Scanning in $z$ was achieved using an objective-mounted piezoelectric Z stage (Physik Instrumente). We used a "fast- $Z$ " mode, where the piezo element would scan the objective focal plane through the sample, return to its initial position at maximum speed, and repeat. Software and a central control board provided by the manufacturer (Thorsync, Thorlabs) were used to synchronize the components and record the state of the imaging and piezo at all times to generate accurate timestamps for the frames.

Scan parameters for the two samples were as follows: For the charged colloidal crystal, a $128 \times 128 \times 251$ pixel box was scanned at 2.17-s intervals. Voxels were cubic, with $0.2-\mu \mathrm{m}$ side length. We chose a spherical region of interest deep inside the interior where the crystal was predominantly monodomain, selecting a cluster of 112 particles to sample over, which was well separated from the sides; for the dense crystalline sediment, a $256 \times 256 \times$ 411 pixel box was scanned at 5.68-s intervals over 1000 
frames. The voxel size is the same as above. A region of interest (ROI) is chosen near the base of the colloidal sediment within which we can track $99 \%$ of all particles, excluding particles at the ROI edge. We studied $n=1481$ particles. Of these, $99.9 \%$ have complete eye trajectories as well.

\section{Particle sizing}

All TPM particles are sized using SEM. The particles are sputter coated with platinum using an SC7620 sputter coater (Quorum Technologies, UK) in an argon atmosphere and imaged at a $10-\mathrm{kV}$ beam energy with a JSM6010LV scanning electron microscopy unit (JEOL, Japan).

\section{ACKNOWLEDGMENTS}

We thank Camille Boulet and Dirk Aarts for assistance with the characterization of the organic solvent mixtures. We also thank Arran Curran for enabling the collection of optical trapping data. T. Y. acknowledges Grants-in-Aid for Young Scientists (B) (No. JP15K17734) and JSPS Fellows (No. JP16J06649) from the Japan Society for the Promotion of Science (JSPS); H. T. acknowledges Grantsin-Aid for Scientific Research (A) (No. JP18H03675) and Specially Promoted Research (No. JP25000002 and No. JP20H05619) from the JSPS; T. Y., Y. L., and R.P. A. D. acknowledge the European Research Council (ERC) (Starting Grant No. 279541-IMCOLMAT and Consolidator Grant No. 724834-OMCIDC).

T. Y., H. T., and R. P. A. D. conceived the project and designed the experiments. T. Y. performed the syntheses, experiments, and analysis. Y.L. prototyped the particles and advised on the experimental work. T. Y. wrote the manuscript; all co-authors discussed and edited the manuscript together. H. T. and R.P. A. D. supervised the project.

\section{APPENDIX A: SYNTHESIS OF OCULI PARTICLES}

\section{Reagents}

We used TPM (Sigma Aldrich), an aqueous ammonium hydroxide solution (28 wt\% solution) (Sigma Aldrich), Cyanine3 NHS Ester (Lumiprobe), BDP-FL NHS Ester (Lumiprobe), anhydrous dimethyl sulfoxide (DMSO) (Sigma Aldrich), 3-aminopropyl trimethoxysilane (APS) (Sigma Aldrich), 4-aminostyrene (Sigma Aldrich), Azobisisobutyronitrile (Sigma Aldrich), and Pluronic F108 (Sigma Aldrich) as received. OLOA stabilizer was provided by Eric Dufresne and Azelis. Double-distilled water was sourced from a Millipore Direct-Q 3 ultrapure water unit.

\section{Preparation of fluorescent monomer}

In order to fluorescently label the TPM particles, we require any fluorophore to bear a reactive component that can be incorporated into the TPM network. TPM has two varieties of reactive moieties, three silyl groups, and a methacrylate group. Both sites are accessible before the final cross-linking step. We proceeded to label the BDPFL NHS Ester with APS, and the Cyanine3 NHS Ester with 4-aminostyrene for incorporation into the silyl and methacrylate networks, respectively. We note that both dyes can react with both labels. For the BDP-FL, $10 \mathrm{mg}$ of reagent was added to $5 \mathrm{~g}$ of chloroform and mixed thoroughly using a PTFE flea and magnetic stirrer. Once the dye was thoroughly dissolved, $10 \mu \mathrm{l}$ of APS (approximately 5 times molar excess) was added to the mixture and allowed to react overnight. For the Cyanine 3 NHS Ester, the same procedure was followed using 4-aminostyrene and DMSO. DMSO was not used for the BDP-FL reaction due to adverse effects on the emission profile. After the reaction, both mixtures could be added directly to TPM particle syntheses.

\section{Synthesis of OCULI particles (charged colloidal crystal)}

We begin by synthesizing the eyes first. The example protocol here describes how the eyes were made for the eyes of the OCULI particles used to make the charged colloidal crystal; it may be modified following previous works [72] to change size and yield. We stirred $40 \mathrm{ml}$ of double-distilled water and $40 \mu \mathrm{l}$ of $28 \mathrm{wt} \%$ ammonium hydroxide solution in a 100-ml round-bottom flask for 15 minutes using an oval PTFE flea. Once a uniform flow was established, $100 \mu \mathrm{l}$ of TPM was introduced to the flask in one smooth motion using a micropipette. It was left to react for approximately 90 minutes. During this time, the TPM hydrolyzed and condensed into monodisperse droplets. Next, $100 \mu \mathrm{l}$ of preprepared chloroform/BDP-FL/APS solution (from above) was added to the mixture. Though the chloroform initially collected at the bottom because of its density and immiscibility, the dye gradually diffused into the mixture and transferred to the TPM phase. After 30 minutes of stirring, approximately $50 \mathrm{mg}$ of AIBN was added, and the mixture was stirred for 20 minutes. It was then transferred to a 50-ml plastic tube and kept in an $80^{\circ} \mathrm{C}$ oven for 3 hours to crosslink the particles. The mixture was subsequently removed and allowed to cool to room temperature. The particles were finally washed at least 5 times using centrifugation (2500 rpm for 5 minutes per spin) to remove unreacted reagents and disperse the particles in water. The final product was resuspended in approximately $1.5 \mathrm{ml}$ of double-distilled water. These particles were approximately $1.0-1.2 \mu \mathrm{m}$ in diameter and notably monodisperse.

Having obtained a suspension of eye particles, we proceeded to coat them with a shell layer to form the 
body of the OCULI particles. This procedure required a solution of prehydrolyzed TPM (hTPM), typically $500 \mu \mathrm{l}$ of TPM stirred in $5 \mathrm{ml}$ of $0.5-\mathrm{mM}$ hydrochloric acid solution. The solution was initially milky but became transparent after approximately 1 hour as the TPM hydrolyzed.

There are two protocol variations for producing OCULI particles. The first is a stepwise addition of hTPM, recommended for making OCULI particles that are less than $2 \mu \mathrm{m}$ in size. The introduction of hTPM by hand is fast and straightforward but entails the risk of nucleating new particles instead of growth on the eye. This is less of a problem for fewer addition steps, i.e., smaller particles, and was used to produce the OCULI particles in the charged colloidal crystal.

An initial basic solution of eye particles was prepared by taking $0.5 \mathrm{ml}$ of the eye solution and adding $5 \mathrm{ml}$ of 14.8-mM ammonium hydroxide. It was kept in a 20-ml glass bottle with a sealed lid. Once the eye particles were well dispersed with a short burst of sonication, $1 \mathrm{ml}$ of the hTPM solution was added, and the bottle quickly tumbled 2 or 3 times. Because of the $\mathrm{pH}$, the hTPM was driven to either form new particles or wet existing ones. The key here was to ensure that there were enough eye particles in the solution to ensure that wetting existing particles occurred at a faster timescale than nucleation but not so many that multiple eyes coalesced into single particles.

After 25 minutes, $1.5 \mathrm{ml}$ of hTPM was dripped into the bottle with gentle manual shaking. The solution was left for another 15 minutes. By this point, the $\mathrm{pH}$ dropped because of dilution, so $1 \mathrm{ml}$ of $14.8-\mathrm{mM}$ ammonium hydroxide was added to compensate. We continued to grow the particles by adding another $1.5 \mathrm{ml}$ of hTPM while shaking, like before. This process was repeated one more time, and the particles were checked using standard optical microscopy.

To dye the particle body, $100 \mu \mathrm{l}$ of Cyanine3/4aminostyrene/DMSO dye was added; the tube was then tumbled and left for 20 minutes. Approximately $50 \mathrm{mg}$ of AIBN was then added before the bottle was tumbled again, left for 20 minutes, then transferred to an oven at $80^{\circ} \mathrm{C}$. The particles were allowed to polymerize for 3 hours, with care taken to manually tumble the bottle every hour to prevent particles from coalescing at the bottom under gravity. After removal and cooling, they were washed using centrifugation (1200 rpm, 5 minutes, 5 times) and redispersed in distilled water.

\section{Synthesis of core-shell OCULI particles (partially crystalline sediment)}

To study dense sediments and jamming, we require particles that are larger and do not suffer the overlap in fluorescence profiles seen in 3D-CSLM with uniformly dyed particles. Thus, we require slightly larger
OCULI and the addition of a nonfluorescent layer at the surface, which is based on the protocol described here [47].

The protocol below was used to create the core-shell OCULI to observe the crystalline sediment. The eyes were produced in a slightly different way; hTPM was prehydrolyzed in a $1: 10$ ratio using a $0.5-\mathrm{mM} \mathrm{HCl}$ solution, as above. Then, $5 \mathrm{ml}$ of this hTPM solution was directly added to $10 \mathrm{ml}$ of $14.8-\mathrm{mM}$ ammonium hydroxide solution in a glass tube before quickly tumbling and leaving stationary on the workbench. Particles could be seen to nucleate within 20 seconds. The solution was left for 20 minutes to allow all of the hTPM to coalesce into droplets. A small PTFE magnetic flea was added, and the mixture was stirred slowly $(<300 \mathrm{rpm})$ to disperse $100 \mu \mathrm{l}$ of the labeled BDP-FL chloroform solution described above and approximately $10 \mathrm{mg}$ of AIBN. After stirring for 20 minutes, the mixture was allowed to polymerize for 3 hours. It was finally redispersed in double-distilled water up to a volume of $10 \mathrm{ml}$ and kept refrigerated until further use.

Having obtained the eyes, we proceeded to make OCULI particles. Here, we follow an alternative method to demonstrate how the size of OCULI may be tailored to different applications, which is particularly recommended for reaching larger particle sizes ( $>2 \mu \mathrm{m}$ in diameter). We added $5 \mathrm{ml}$ of $14.8-\mathrm{mM}$ ammonium hydroxide to a $15-\mathrm{ml}$ plastic tube, followed by $1 \mathrm{ml}$ of the suspension of particles prepared above. The solution was sonicated for 10 minutes to ensure that no clusters became encapsulated instead of single particles, followed by $1 \mathrm{ml}$ of a freshly prepared $1: 10$ hTPM solution before the tube was tumbled and left stationary for 30 minutes. This suspension was then added to $20 \mathrm{ml}$ of $14.8-\mathrm{mM}$ ammonium hydroxide solution in a 50 -ml round-bottom flask with a magnetic flea. The mixture was stirred slowly $(<100 \mathrm{rpm})$ to ensure homogenization while preventing the particles from coalescing. Then, $10 \mathrm{ml}$ of hTPM solution was introduced dropwise over 2 hours using a 10-ml plastic syringe, a PTFE tube, and a syringe pump. We note here that the preaddition of $1 \mathrm{ml}$ of hTPM, followed by a further dropwise addition, led to monodisperse particles, while the direct dropwise addition of hTPM to cores did not. This result may be due to an energetic barrier to prewetting the hTPM, which is overcome for different particles at different times given a low concentration of hTPM; this issue was solved by introducing a higher concentration of hTPM initially, followed by the gradual addition of the rest of the material.

If standard OCULI particles are required, $100 \mu \mathrm{l}$ of dye solution and $20 \mathrm{mg}$ of AIBN should be added to the mixture before stirring for 20 minutes and then heated in an $80^{\circ} \mathrm{C}$ oven for 2 hours. However, since the objective of this synthesis was to produce core-shell OCULI, $4 \mathrm{ml}$ of $5 \mathrm{wt} \%$ Pluronic F108 solution was added, and the mixture was stirred for 20 minutes before 
adding the dye/AIBN and heating for 2 hours. This process is required to modify the wetting properties of the particles for the next step.

Once these OCULI were cross-linked, they were washed thoroughly using centrifugation and double-distilled water (1000 $g$ spins, 10 minutes). Any smaller particles left in the solution at this stage would lead to large secondary particles growing in the following steps. The final particle solution was dispersed thoroughly into double-distilled water up to a total volume of $10 \mathrm{ml}$, which, again, may be refrigerated until further use.

We proceeded to add the nonfluorescent TPM layer. We began by nucleating droplets on the surface of the OCULI to produce "raspberry"-shaped OCULI. We added $2 \mathrm{ml}$ of the OCULI solution above to $100 \mathrm{ml}$ of $14.8-\mathrm{mM}$ ammonia solution in a $250-\mathrm{ml}$ plastic container. We added $2 \mathrm{ml}$ of freshly prepared hTPM before the container was quickly tumbled and left stationary for 45 minutes. Then, $20 \mathrm{mg}$ of AIBN was added before the container was transferred to an $80^{\circ} \mathrm{C}$ oven and left for 3 hours (note that the volume is greater, so the solution requires more time to reach the target temperature). After the added hTPM was cross-linked, the particles were again washed thoroughly with double-distilled water using centrifugation $(1000 \mathrm{~g}, 10$ minutes, 5 times) until any particles nucleated in the bulk were removed. The original OCULI were covered in a dense layer of "lobes," as seen in Figs. 1(b3) and 1(c3) in the main text. The final suspension was redispersed into double-distilled water with a total volume of $2 \mathrm{ml}$, the same volume as the solution of OCULI that was added.

Finally, we filled in the space between the lobes to create smooth, spherical colloidal particles. The $2 \mathrm{ml}$ of raspberry OCULI were again added to $100 \mathrm{ml}$ of $14.8-\mathrm{mM}$ ammonia solution in a $250-\mathrm{ml}$ plastic container. This was followed by $2 \mathrm{ml}$ of freshly prepared hTPM solution before the container was quickly tumbled and left stationary for 60 minutes. The appearance of the particles was quickly confirmed under bright-field microscopy, with particular attention to how circular the diffraction-limited rings surrounding the particles looked. The particles were still rough, so another $2 \mathrm{ml}$ of hTPM was added before tumbling and waiting for another 60 minutes. This process was repeated as many times as required (in this case, a total of 4 times, including the first addition) to obtain spherical particles. Under bright-field microscopy, the particles at this point did not appear perfectly spherically symmetric due to the index mismatch between the cross-linked lobes and the un-cross-linked TPM wetting the gaps between them. The best indicator was the shape of the diffraction rings around the particle. Following the hTPM addition, $20 \mathrm{mg}$ of AIBN was added before the container was transferred to an $80^{\circ} \mathrm{C}$ oven and heated for 3 hours. The mixture was then recovered and allowed to cool to room temperature before the particles were cleaned again with double-distilled water and centrifugation $(1000 \mathrm{~g}, 5$ minutes, 5 times). Finally, the sample was again redispersed into $2 \mathrm{ml}$ of double-distilled water and was then ready for use in experiments.

Note that the procedure to transfer these particles from water to organic solvent mixtures is detailed in Ref. [48].

\section{APPENDIX B: ROTATIONAL DIFFUSION CONSTANT AND THE AUTOCORRELATION OF THE UNIT VECTOR}

This derivation is largely reproduced from Ref. [2]. For a spherical particle, the rotational diffusion constant $D_{r}$ is defined as the rate at which an arbitrary point fixed to the surface diffuses while confined to the surface. Given a reference axis that runs from the center of the sphere to the point, we consider the probability that the point is located at point $P(\theta, \phi)$ in spherical coordinates at some time $t$. We may first define a diffusion equation based on this definition,

$$
\frac{\partial}{\partial t} P(\theta, \phi, t)=D_{r} \nabla^{2} P(\theta, \phi, t)
$$

where $P(\theta, \phi, t)$ is the probability density function that the point will be located at point $(\theta, \phi)$ after a time $t$ in spherical coordinates. This formulation is particularly well suited for measurements using the OCULI, where the eye defines a fixed point on the surface. The diffusion of the point matches the time evolution of the unit orientation vector $\boldsymbol{u}(t)$. The time-averaged autocorrelation of the orientation vector $\boldsymbol{u}$ may thus be expressed as

$$
\left\langle\mathbf{u}\left(t_{0}\right) \cdot \mathbf{u}\left(t_{0}+t\right)\right\rangle=\langle\cos \theta(t)\rangle .
$$

Here, we assume that the orientation vector is oriented along the $z$-axis at $t_{0}$, so the function $P(\theta, \phi, t)$ becomes independent of $\phi$. Thus, $P(\theta, \phi)=P(\theta)$. The autocorrelation $\langle\cos \theta\rangle$ may now be expressed using $P(\theta, t)$ as follows:

$$
\begin{aligned}
\langle\cos \theta\rangle & =\int d \theta d \phi P(\theta) \cos \theta \sin \theta \\
& =2 \pi \int_{0}^{\pi} d \theta P(\theta) \cos \theta \sin \theta .
\end{aligned}
$$

Using the diffusion equation above, the time derivative of this expectation value is then given by

$$
\begin{aligned}
\frac{\partial}{\partial t}\langle\cos \theta\rangle & =2 \pi \int_{0}^{\pi} d \theta \frac{\partial P(\theta)}{\partial t} \cos \theta \sin \theta \\
& =2 \pi D_{r} \int_{0}^{\pi} d \theta\left(\nabla^{2} P(\theta)\right) \cos \theta \sin \theta .
\end{aligned}
$$


Reexpressing the $\nabla^{2}$ operator in spherical coordinates, we apply two partial integrations and find

$$
\begin{aligned}
\frac{\partial}{\partial t}\langle\cos \theta\rangle & =-2 D_{r} \int_{0}^{\pi} d \theta P(\theta) \sin \theta \cos \theta \\
& =-2 D_{r}\langle\cos \theta\rangle,
\end{aligned}
$$

which shows that the time-averaged autocorrelation of the unit orientation vector $\langle\cos \theta\rangle$ has an exponential decay, where

$$
\langle\cos \theta\rangle=e^{-2 D_{r} t}
$$

\section{APPENDIX C: ROTATIONAL DIFFUSION IN ULTRADILUTE SUSPENSIONS}

For the core-shell system, we considered the diffusion constant of these particles in the ultradilute limit; an accurate benchmark was required to quantitatively characterize diffusion constants as a function of local volume fraction. To do this, we modified the density of the solvent slightly, using a 4:1-by-volume mixture of TCE and tetralin, to prevent sedimentation and accumulation of the particles at the base of the microscopy cell. We assumed the slight change in the composition of the solvent did not adversely affect the particle size. We accounted for a change in shear viscosity $(1.43 \mathrm{mPa} \cdot \mathrm{s})$. Sampling trajectories away from surfaces, the autocorrelation of the particle orientations is shown in Fig. 5, yielding a relaxation time of $\tau_{r}=16.9 \pm 0.2 \mathrm{~s}$. This may be converted to a diffusivity and then an effective hydrodynamic diameter using the equation $D_{r}=k_{\mathrm{B}} T / \pi \eta \sigma_{H}^{3}$; we found that $\sigma_{H}=3.14 \mu \mathrm{m}$. This result is consistent with the size found by SEM, noting that both particle shrinkage due to drying for SEM imaging and swelling in the haloalkane solvent contribute to making the SEM value smaller. Thus, we

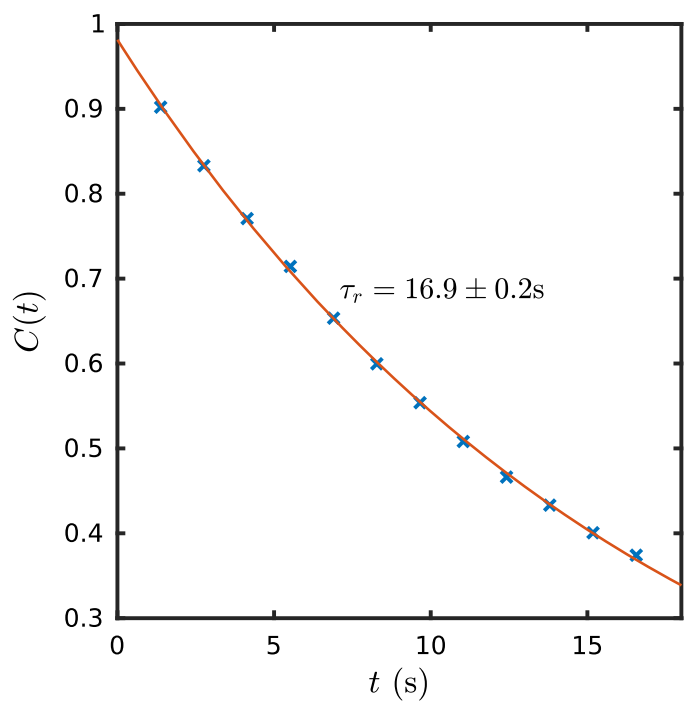

FIG. 5. Orientation autocorrelation $C(t)$ for core-shell OCULI in the ultradilute limit. Here, $C(t)$ is for the same core-shell OCULI particles used for the dense crystalline sediment. The data fit well with an exponential decay (red solid curve).

can also calculate an expansion coefficient linking the SEM size to a hydrodynamic diameter of $10 \%$.

\section{APPENDIX D: CALCULATION OF THE ROTATIONAL COUPLING CONSTANT $\boldsymbol{R}_{C}$}

In order to characterize the coupling between the rotation of different pairs of particles, we calculate the rotational coupling constant $R_{C}$ from an estimate of the angular velocity vector $\boldsymbol{\omega}$ over time. Here, $\boldsymbol{\omega}$ is estimated from how the unit orientation vector changes from $\boldsymbol{u}(t)$ to $\boldsymbol{u}(t+\Delta t)$, where $\Delta t$ is the time between consecutive frames. Thus, $R_{C}$ may be expressed using $\boldsymbol{u}(t)$ as follows:

$$
R_{c}(\tau, r)=\frac{\boldsymbol{\omega}_{i} \cdot \boldsymbol{\omega}_{j}}{\left\langle\left|\boldsymbol{\omega}_{i}^{2}\right|\right\rangle^{1 / 2}\left\langle\left|\boldsymbol{\omega}_{j}^{2}\right|\right\rangle^{1 / 2}} \approx \frac{\left\langle\left[\boldsymbol{u}_{i}\left(r_{i j}, \tau\right) \otimes \boldsymbol{u}_{i}\left(r_{i j}, 0\right)\right] \cdot\left[\boldsymbol{u}_{j}\left(r_{i j}, \tau\right) \otimes \boldsymbol{u}_{j}\left(r_{i j}, 0\right)\right]\right\rangle}{\left\langle\left|\boldsymbol{u}_{i}\left(r_{i j}, \tau\right) \otimes \boldsymbol{u}_{i}\left(r_{i j}, 0\right)\right|^{2}\right\rangle^{1 / 2}\left\langle\left|\boldsymbol{u}_{j}\left(r_{i j}, \tau\right) \otimes \boldsymbol{u}_{j}\left(r_{i j}, 0\right)\right|^{2}\right\rangle^{1 / 2}}
$$

\section{APPENDIX E: COMPARISON WITH DENSITY-MATCHED SCENARIOS FOR CHARGED CRYSTALS}

The single-particle rotational diffusivity seen in the charged crystal is slower than what is expected in the ultradilute limit using the particle size found from SEM, $\tau_{0}=1 / 2 D_{0}=2.26 \mathrm{~s}$, which cannot be simply accounted for by swelling of the particle; using an experimentally determined expansion ratio from SEM sizes to hydrodynamic diameters (see Appendix C), $\tau_{0}=3.01 \mathrm{~s}$. Previous work $[37,46,73]$ has determined analytical forms for how rotational diffusivity changes with volume fraction. Adopting the expression used here [37] (also tested against numerical simulations in Ref. [58]), $D_{r}=D_{0}\left(1-0.63 \phi-0.67 \phi^{2}\right), \tau_{r}=3.47 \mathrm{~s}$, which is still shorter than the $\tau_{r}=5.8 \pm 0.1 \mathrm{~s}$ found in our experiment. 


\section{APPENDIX F: CONFIRMATION OF HARD-SPHERE-LIKE INTERPARTICLE INTERACTIONS}

To ensure that hard-sphere-like interactions were realized in 5 wt\% OLOA 11000, an optical trapping experiment was carried out to confirm the lack of any long-ranged repulsive interactions. For the identical solvent used for the semicrystalline sediment (3:1 TCE:TET, 5 wt\% OLOA 11000), we applied the same optical trapping methodology as in Ref. [48] to directly characterize the interaction potential. Specifically, we trapped two core-shell OCULI particles (SEM diameter $4.01 \mu \mathrm{m}, 3.5 \%$ polydispersity) in two weak optical potentials, moved the potentials to different distances from each other $\left(r_{\text {trap }}\right)$, and measured the interparticle separation $\left(r_{p}\right)$ as a measure of repulsive force. Any long-ranged repulsive interaction will appear as a deviation in the interparticle separations from the separation between the traps.

First, we confirm that the traps are weak enough to allow measurement of significant deviations at low energetic cost. The confining potential is found from the probability distribution $p(r)$ of the fluctuating interparticle separation $r$ by a simple inversion, $\beta U(\Delta r)=-k_{B} T \log [p(\Delta r)]$, where $\Delta r=r-\langle r\rangle$, as given in the inset of Fig. 6; we confirm that the confinement is harmonic to a good

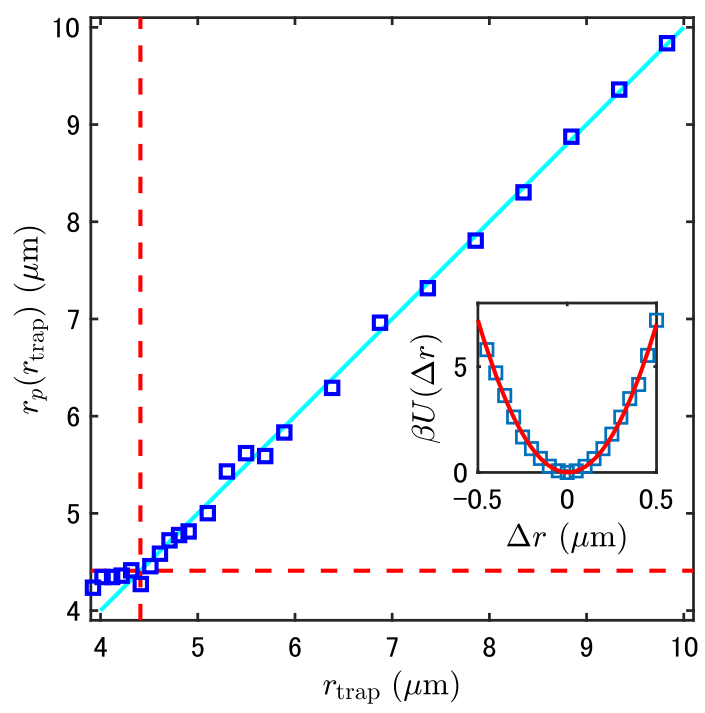

FIG. 6. Direct observation of lack of long-ranged interactions in the hard-sphere system. We show the optical trapping measurement of the interaction between a pair of core-shell OCULI particles in a pair of weak optical traps at different separations. There is no deviation between interparticle separation $r_{p}$ and trap positions $r_{\text {trap }}$ until the particles meet, at which point the interparticle separation becomes constant. This case is consistent with a hard-spherelike separation. Inset: estimate of the optical confinement potential $U(\Delta r)$. approximation (fit) and show that an interaction energy of $k_{B} T$ would cause deviations in excess of $100 \mathrm{~nm}$, which is easily observable. Now, we look at how the interparticle separations change as the traps are brought closer together. Going from large to small separations, when a deviation is finally observed, the interparticle separation basically remains constant (see Fig. 6). Red dashed lines show the average interparticle separation at the three smallest trap separations, $4.31 \mu \mathrm{m}$, consistent with $4.01 \mu \mathrm{m}$ under SEM swelled in the solvent.

Both our own experimental data and evidence from the literature as introduced in the main text point to negligible surface charge and strong screening in this dense colloidal system. We may safely conclude that the interparticle repulsion in this system is hard-sphere-like. In hindsight, this conclusion also explains the agreement in our sediment between the particle diameter found via SEM $(2.81 \mu \mathrm{m})$ and the effective hard-sphere size found from phase coexistence $(3.00 \mu \mathrm{m})$. See Appendix G for details.

\section{APPENDIX G: STATIC STRUCTURE IN A PARTIALLY CRYSTALLINE SEDIMENT OF CORE-SHELL OCULI}

Here, we describe the static structure of the dense crystalline sediment. We first note that there is a small fraction of particles that fused together during the synthesis, when raspberry particles are formed. These particles are easily separable by considering the minimum distance to a neighbor and the arrest of rotational motion, as shown in Fig. 7(a). Particles where $d_{\mathrm{NN}}<$ 2.5 and $C\left(3 \tau_{0}\right)>0.9$ are excluded from all analyses. We also consider a broad variation in density over the sample. A Voronoi tessellation allows us to calculate the local number density $n$ for all particles, averaging over 100 frames to lessen the effect of thermal fluctuations. A profile of $n$ with height $z$ is given as an inset in Fig. 7(b). Note that there is a small reduction with height, but this is very small, which is in agreement with theoretical predictions for the density profile away from the top sediment interface [74]. We also see that $n$ for particles above and below the median height have similar distributions [see Fig. 7(b)], with a similarly wide range.

The system is partially crystalline. We may characterize this by considering the bond-orientational order $q_{6}$ [75], which is widely accepted as an appropriate local order parameter for crystallization in hard-sphere-like systems [76,77]. To associate nearest neighbors, we use a simple distance threshold taken from the first minimum of the radial distribution function $g(r)$ in Fig. 7(c). We consider the distribution of $q_{6}$, shown in Fig. 7(d). One can clearly see that a boundary $q_{6} \approx 0.5$ separates crystalline and noncrystalline parts of the sample. We may use this threshold and the Voronoi tessellation above to find the proportion of the sample that is crystalline. The sum of the 

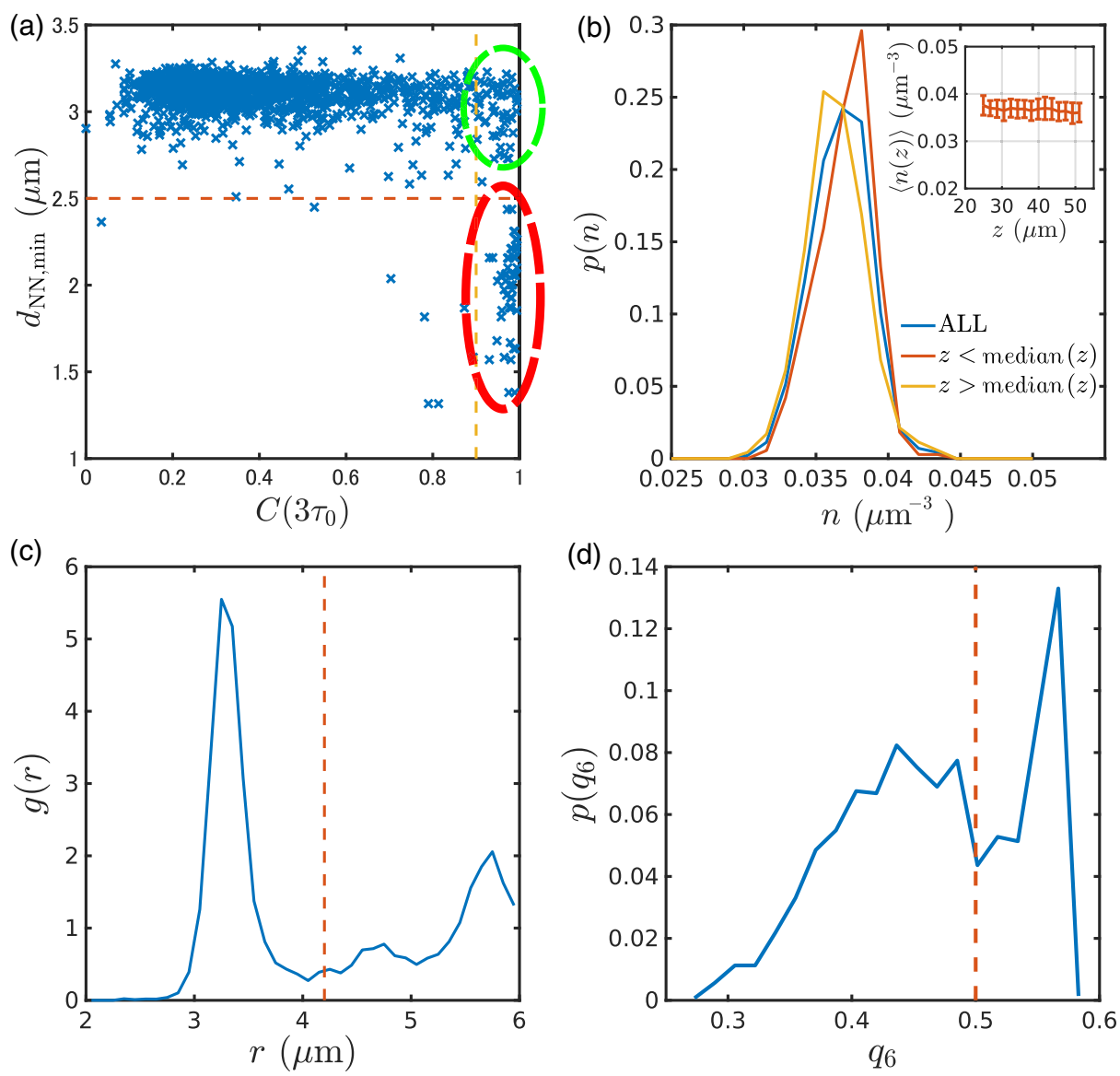

FIG. 7. Static properties of dense, partially crystalline sediment of core-shell OCULI particles. (a) Minimum distance to neighboring particles as a function of $C\left(3 \tau_{0}\right)$. Particles arrested by frictional interactions are indicated by the upper green dashed circle. Particles arrested due to dimerization with other particles during the growth of the shell are shown by the lower red dashed circle. These particles are omitted from the analysis. (b) Histogram of local volume fraction $\phi$ for particles below and above the median $z$ position, as well as the distribution for all particles in the ROI. There is a small variation, but both populations contain a wide range of $\phi$. Inset: average $\phi$ as a function of $z$. There is a gentle decrease with height, as expected in a sediment, but the variation is very small. (c) Radial distribution function. The threshold used for detecting nearest neighbors is given as a dashed line. (d) Distribution of $q_{6}$ over the whole ROI. The threshold used to separate crystalline and noncrystalline particles is given as a dashed line.

volume associated with particles with $q_{6}>0.5$ is $34.3 \%$ of the total volume of the ROI. Assuming that the sample is monodisperse, we may use this and the average number density to calculate an effective diameter for the particles,

$$
\sigma_{\mathrm{HS}}=\left(\frac{6}{\phi_{\mathrm{HS}} \pi n_{\mathrm{tot}}}\right)^{\frac{1}{3}}
$$

For the sample shown, $\sigma_{\mathrm{HS}}=3.00 \mu \mathrm{m}$. We use this and local volumes found from Voronoi tessellation to calculate local volume fractions $\phi_{i}$ in the main text of the paper.

\section{APPENDIX H: LOCAL VOLUME FRACTION VS CLOSEST DISTANCE TO NEAREST-NEIGHBOR PARTICLE}

To rationalize the relationship between the rotational diffusivity and the volume fraction, we find the distance to

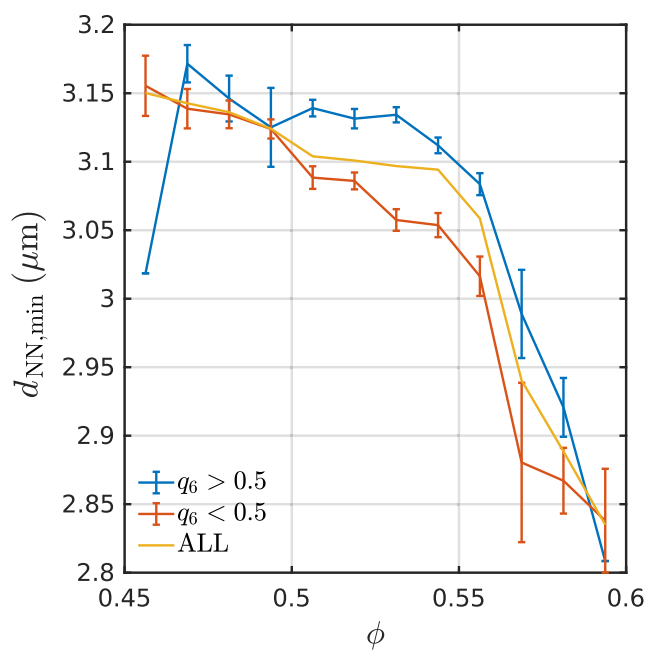

FIG. 8. Distance to the closest nearest neighbor $d_{\mathrm{NN}, \mathrm{min}}$ as a function of volume fraction $\phi$ for particles with different crystallinity, as indicated. 

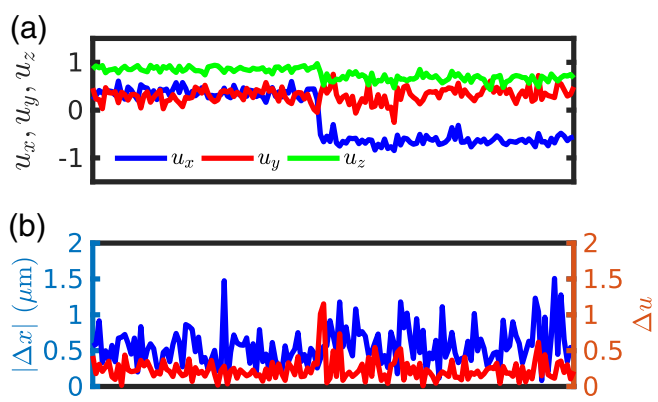

(c)

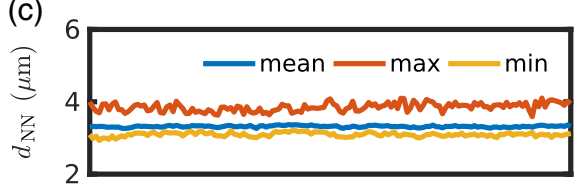

(d)

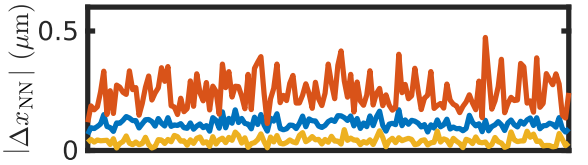

(e)

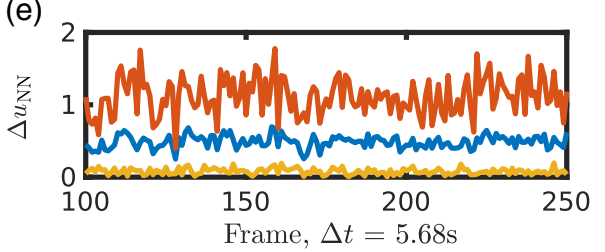

FIG. 9. Intermittent dynamics and neighboring particles. (a) $x$, $y$, and $z$ components of the unit orientation vector of a particle undergoing stick-slip rotational motion as a function of time. All data below refer to the same trajectory. (b) Movement of the same particle over two frames, both translational $\Delta x$ and orientational $\Delta u$ displacements. (c) Distance to nearest neighbors $d_{\mathrm{NN}}$ as a function of time. (d) Displacements of nearest neighbors over two frames as a function of time. (e) Displacements in orientation $\Delta u$ of nearest neighbors over two frames as a function of time. (c-e) show the mean, maximum and minimum for each statistic.

the closest nearest neighbor $d_{\min }$ for the same particle populations in each volume fraction $\phi$ bin, as shown in Fig. 8. It is clear that particles with higher crystallinity have a greater $d_{\min }$ than those with lower crystallinity for the volume fraction range where trends in $D_{r} / D_{0}$ change. Looking closely, we note that the deviation between crystalline and noncrystalline particles starts at a lower $\phi$ than for $D_{r} / D_{0}$. At lower $\phi$, the particles are not close enough together to strongly couple hydrodynamically; thus, the effect only arises at higher $\phi$.

\section{APPENDIX I: STICK-SLIP DYNAMICS AND DYNAMICS OF NEIGHBORING PARTICLES}

On observing intermittent rotational dynamics of a subpopulation of particles, we considered whether this was simply correlated with a fluctuation in the position or orientation of the particle itself, or its neighbors, which is not the case. As shown in Fig. 9, we can see that the intermittent rotational slip of the particle itself (a) is not accompanied by the displacement of the particle itself (b), nor the distance to (c), displacement of (d), or rotation of (e) its nearest neighbors.

[1] W. C. K. Poon, Colloids as Big Atoms, Science 304, 830 (2004).

[2] A. P. Philipse, Brownian Motion, Undergraduate Lecture Notes in Physics (Springer International Publishing, Cham, 2018).

[3] T. Palberg, Crystallization Kinetics of Colloidal Model Suspensions: Recent Achievements and New Perspectives, J. Phys. Condens. Matter 26, 333101 (2014).

[4] J. Russo and H. Tanaka, The Microscopic Pathway to Crystallization in Supercooled Liquids, Sci. Rep. 2, 505 (2012).

[5] S. Arai and H. Tanaka, Surface-Assisted Single-Crystal Formation of Charged Colloids, Nat. Phys. 13, 503 (2017).

[6] Y.L. Wu, D. Derks, A. van Blaaderen, and A. Imhof, Melting and Crystallization of Colloidal Hard-Sphere Suspensions under Shear, Proc. Natl. Acad. Sci. U.S.A. 106, 10564 (2009).

[7] Z. Wang, F. Wang, Y. Peng, Z. Zheng, and Y. Han, Imaging the Homogeneous Nucleation During the Melting of Superheated Colloidal Crystals, Science 338, 87 (2012).

[8] P. J. Lu, E. Zaccarelli, F. Ciulla, A. B. Schofield, F. Sciortino, and D. A. Weitz, Gelation of Particles with Short-Range Attraction, Nature (London) 453, 499 (2008).

[9] M. Tateno and H. Tanaka, Numerical Prediction of Colloidal Phase Separation by Direct Computation of NavierStokes Equation, npj Comput. Mater. 5, 40 (2019).

[10] G. L. Hunter and E. R. Weeks, The Physics of the Colloidal Glass Transition, Rep. Prog. Phys. 75, 066501 (2012).

[11] P. J. Lu and D. A. Weitz, Colloidal Particles: Crystals, Glasses, and Gels, Annu. Rev. Condens. Matter Phys. 4, 217 (2013).

[12] J. B. Perrin, Les Atomes (Félix Alcan, Paris, 1913).

[13] A. Furukawa and H. Tanaka, Key Role of Hydrodynamic Interactions in Colloidal Gelation, Phys. Rev. Lett. 104, 245702 (2010).

[14] Z. Varga, G. Wang, and J. Swan, The Hydrodynamics of Colloidal Gelation, Soft Matter 11, 9009 (2015).

[15] J. De Graaf, W. C. K. Poon, M. J. Haughey, and M. Hermes, Hydrodynamics Strongly Affect the Dynamics of Colloidal Gelation but Not Gel Structure, Soft Matter 15, 10 (2019).

[16] N. J. Wagner and J. F. Brady, Shear Thickening in Colloidal Dispersions, Phys. Today 62, No. 10, 27 (2009).

[17] J. J. Stickel and R. L. Powell, Fluid Mechanics and Rheology of Dense Suspensions, Annu. Rev. Fluid Mech. 37, 129 (2005).

[18] T. Ando and J. Skolnick, Crowding and Hydrodynamic Interactions Likely Dominate In Vivo Macromolecular Motion, Proc. Natl. Acad. Sci. U.S.A. 107, 18457 (2010). 
[19] R. Golestanian, J. M. Yeomans, and N. Uchida, Hydrodynamic Synchronization at Low Reynolds Number, Soft Matter 7, 3074 (2011).

[20] M. A. Mourão, J. B. Hakim, and S. Schnell, Connecting the Dots: The Effects of Macromolecular Crowding on Cell Physiology, Biophys. J. 107, 2761 (2014).

[21] Y. Wang, Y. Wang, X. Zheng, E. Ducrot, J. S. Yodh, M. Weck, and D. J. Pine, Crystallization of DNA-Coated Colloids, Nat. Commun. 6, 7253 (2015).

[22] E. Brown and H. M. Jaeger, Shear Thickening in Concentrated Suspensions: Phenomenology, Mechanisms and Relations to Jamming, Rep. Prog. Phys. 77, 046602 (2014).

[23] X. Cheng, J. H. McCoy, J. N. Israelachvili, and I. Cohen, Imaging the Microscopic Structure of Shear Thinning and Thickening Colloidal Suspensions, Science 333, 1276 (2011).

[24] N. Y. C. Lin, B. M. Guy, M. Hermes, C. Ness, J. Sun, W. C. K. Poon, and I. Cohen, Hydrodynamic and Contact Contributions to Continuous Shear Thickening in Colloidal Suspensions, Phys. Rev. Lett. 115, 228304 (2015).

[25] N. Y. C. Lin, C. Ness, M. E. Cates, J. Sun, and I. Cohen, Tunable Shear Thickening in Suspensions, Proc. Natl. Acad. Sci. U.S.A. 113, 10774 (2016).

[26] J. R. Royer, D. L. Blair, and S. D. Hudson, Rheological Signature of Frictional Interactions in Shear Thickening Suspensions, Phys. Rev. Lett. 116, 188301 (2016).

[27] J. Comtet, G. Chatté, A. Niguès, L. Bocquet, A. Siria, and A. Colin, Pairwise Frictional Profile between Particles Determines Discontinuous Shear Thickening Transition in Non-colloidal Suspensions, Nat. Commun. 8, 15633 (2017).

[28] P. Sehgal, M. Ramaswamy, I. Cohen, and B. J. Kirby, Using Acoustic Perturbations to Dynamically Tune Shear Thickening in Colloidal Suspensions, Phys. Rev. Lett. 123, 128001 (2019).

[29] N. Fernandez, R. Mani, D. Rinaldi, D. Kadau, M. Mosquet, H. Lombois-Burger, J. Cayer-Barrioz, H. J. Herrmann, N. D. Spencer, and L. Isa, Microscopic Mechanism for Shear Thickening of Non-Brownian Suspensions, Phys. Rev. Lett. 111, 108301 (2013).

[30] M. Wyart and M. E. Cates, Discontinuous Shear Thickening without Inertia in Dense Non-Brownian Suspensions, Phys. Rev. Lett. 112, 098302 (2014).

[31] R. Mari, R. Seto, J. F. Morris, and M. M. Denn, Discontinuous Shear Thickening in Brownian Suspensions by Dynamic Simulation, Proc. Natl. Acad. Sci. U.S.A. 112, 15326 (2015).

[32] S. Jamali and J. F. Brady, Alternative Frictional Model for Discontinuous Shear Thickening of Dense Suspensions: Hydrodynamics, Phys. Rev. Lett. 123, 138002 (2019).

[33] M. Ramaswamy, N. Y. C. Lin, B. D. Leahy, C. Ness, A. M. Fiore, J. W. Swan, and I. Cohen, How Confinement-Induced Structures Alter the Contribution of Hydrodynamic and Short-Ranged Repulsion Forces to the Viscosity of Colloidal Suspensions, Phys. Rev. X 7, 041005 (2017).

[34] Z. Dogic and S. Fraden, Ordered Phases of Filamentous Viruses, Curr. Opin. Colloid Interface Sci. 11, 47 (2006).

[35] P. B. Muller, M. Rossi, A. G. Marín, R. Barnkob, P. Augustsson, T. Laurell, C. J. Kähler, and H. Bruus, Ultrasound-Induced Acoustophoretic Motion of Microparticles in Three Dimensions, Phys. Rev. E 88, 023006 (2013).
[36] I. I. Smalyukh, Liquid Crystal Colloids, Annu. Rev. Condens. Matter Phys. 9, 207 (2018).

[37] V. Degiorgio, R. Piazza, and R. B. Jones, Rotational Diffusion in Concentrated Colloidal Dispersions of Hard Spheres, Phys. Rev. E 52, 2707 (1995).

[38] G. H. Koenderink, M. P. Lettinga, and A. P. Philipse, Rotational Dynamics of Charged Colloidal Spheres: Role of Particle Interactions, J. Chem. Phys. 117, 7751 (2002).

[39] G. H. Koenderink, D. G. A. L. Aarts, and A. P. Philipse, Rotational Dynamics of Colloidal Tracer Spheres in Suspensions of Charged Rigid Rods, J. Chem. Phys. 119, 4490 (2003).

[40] M. Reichert and H. Stark, Hydrodynamic Coupling of Two Rotating Spheres Trapped in Harmonic Potentials, Phys. Rev. E 69, 031407 (2004).

[41] C. J. Behrend, J. N. Anker, B. H. McNaughton, and R. Kopelman, Microrheology with Modulated Optical Nanoprobes (MOONs), J. Magn. Magn. Mater. 293, 663 (2005).

[42] M. P. Lettinga, G. H. Koenderink, B. W. M. Kuipers, E. Bessels, and A. P. Philipse, Rotational Dynamics of Colloidal Spheres Probed with Fluorescence Recovery after Photobleaching, J. Chem. Phys. 120, 4517 (2004).

[43] J. Wenzl, R. Seto, M. Roth, H. J. Butt, and G. K. Auernhammer, Measurement of Rotation of Individual Spherical Particles in Cohesive Granulates, Granular Matter 15, 391 (2013).

[44] B. Ilhan, J. J. Schoppink, F. Mugele, and M. H. G. Duits, Spherical Probes for Simultaneous Measurement of Rotational and Translational Diffusion in 3 Dimensions, J. Colloid Interface Sci. 576, 322 (2020).

[45] B. Liu and A. Böker, Measuring Rotational Diffusion of Colloidal Spheres with Confocal Microscopy, Soft Matter 12, 6033 (2016).

[46] S. Schütter, J. Roller, A. Kick, J.-M. Meijer, and A. Zumbusch, Real-Space Imaging of Translational and Rotational Dynamics of Hard Spheres from the Fluid to the Crystal, Soft Matter 13, 8240 (2017).

[47] Y. Liu, K. V. Edmond, A. Curran, C. Bryant, B. Peng, D. G. A. L. Aarts, S. Sacanna, and R. P. A. Dullens, CoreShell Particles for Simultaneous 3D Imaging and Optical Tweezing in Dense Colloidal Materials, Adv. Mater. 28, 8001 (2016).

[48] Y. Liu, T. Yanagishima, A. Curran, K. V. Edmond, S. Sacanna, and R.P.A. Dullens, Colloidal Organosilica Spheres for Three-Dimensional Confocal Microscopy, Langmuir 35, 7962 (2019).

[49] V. N. Manoharan, Pinned Down, Nat. Mater. 14, 869 (2015).

[50] M. Kamp, B. de Nijs, J. J. Baumberg, and O. A. Scherman, Contact Angle as a Powerful Tool in Anisotropic Colloid Synthesis, J. Colloid Interface Sci. 581, 417 (2021).

[51] M. Leocmach and H. Tanaka, A Novel Particle Tracking Method with Individual Particle Size Measurement and Its Application to Ordering in Glassy Hard Sphere Colloids, Soft Matter 9, 1447 (2013).

[52] R. P. A. Dullens, M. Claesson, D. Derks, A. Van Blaaderen, and W. K. Kegel, Monodisperse Core-Shell Poly (Methyl Methacrylate) Latex Colloids, Langmuir 19, 5963 (2003). 
[53] T. E. Kodger, R. E. Guerra, and J. Sprakel, Precise Colloids with Tunable Interactions for Confocal Microscopy, Sci. Rep. 5, 14635 (2015).

[54] J. C. Crocker and D. G. Grier, Methods of Digital Video Microscopy for Colloidal Studies, J. Colloid Interface Sci. 179, 298 (1996).

[55] J. Santana-Solano, D. T. Wu, and D. W. M. Marr, Direct Measurement of Colloidal Particle Rotation and Field Dependence in Alternating Current Electrohydrodynamic Flows, Langmuir 22, 5932 (2006).

[56] Y. Goto and H. Tanaka, Purely Hydrodynamic Ordering of Rotating Disks at a Finite Reynolds Number, Nat. Commun. 6, 5994 (2015).

[57] K. Han, G. Kokot, S. Das, R. G. Winkler, G. Gompper, and A. Snezhko, Reconfigurable Structure and Tunable Transport in Synchronized Active Spinner Materials, Sci. Adv. 6, eaaz8535 (2020).

[58] M. H. J. Hagen, D. Frenkel, and C. P. Lowe, Rotational Diffusion in Dense Suspensions, Physica (Amsterdam) 272A, 376 (1999).

[59] A. Socoliuc, R. Bennewitz, E. Gnecco, and E. Meyer, Transition from Stick-Slip to Continuous Sliding in Atomic Friction: Entering a New Regime of Ultralow Friction, Phys. Rev. Lett. 92, 134301 (2004).

[60] A. Vanossi, N. Manini, M. Urbakh, S. Zapperi, and E. Tosatti, Colloquium: Modeling Friction: From Nanoscale to Mesoscale, Rev. Mod. Phys. 85, 529 (2013).

[61] H. M. Jaeger, C. H. Liu, S. R. Nagel, and T. A. Witten, Friction in Granular Flows, Europhys. Lett. 11, 619 (1990).

[62] A. Fall, F. Bertrand, G. Ovarlez, and D. Bonn, Yield Stress and Shear Banding in Granular Suspensions, Phys. Rev. Lett. 103, 178301 (2009).

[63] M. P. Ciamarra, R. Pastore, M. Nicodemi, and A. Coniglio, Jamming Phase Diagram for Frictional Particles, Phys. Rev. E 84, 041308 (2011).

[64] K. M. Salerno, D. S. Bolintineanu, G. S. Grest, J. B. Lechman, S. J. Plimpton, I. Srivastava, and L. E. Silbert, Effect of Shape and Friction on the Packing and Flow of Granular Materials, Phys. Rev. E 98, 050901(R) (2018).

[65] H. Yoshizawa and J. Israelachvili, Fundamental Mechanisms of Interfacial Friction. 2. Stick-Slip Friction of Spherical and Chain Molecules, J. Phys. Chem. 97, 11300 (1993).
[66] T. Palberg and K. Streicher, Resonant Stick-Slip Motion in a Colloidal Crystal, Nature (London) 367, 51 (1994).

[67] C. Ligoure and L. Leibler, Thermodynamics and Kinetics of Grafting End-Functionalized Polymers to an Interface, J. Phys. 51, 1313 (1990).

[68] S. Yasin, Ph.D. thesis, Imperial College, London (2011).

[69] G. T. Hermanson, Bioconjugate Techniques, 2nd ed. (Elsevier, New York, 2013).

[70] R. Seto, R. Mari, J. F. Morris, and M. M. Denn, Discontinuous Shear Thickening of Frictional Hard-Sphere Suspensions, Phys. Rev. Lett. 111, 218301 (2013).

[71] L. C. Hsiao and S. Pradeep, Experimental Synthesis and Characterization of Rough Particles for Colloidal and Granular Rheology, Curr. Opin. Colloid Interface Sci. 43, 94 (2019).

[72] C. Van der Wel, R. K. Bhan, R. W. Verweij, H. C. Frijters, Z. Gong, A. D. Hollingsworth, S. Sacanna, and D. J. Kraft, Preparation of Colloidal Organosilica Spheres through Spontaneous Emulsification, Langmuir 33, 8174 (2017).

[73] H. J. H. Clercx and P. P. J. M. Schram, Three Particle Hydrodynamic Interactions in Suspensions, J. Chem. Phys. 96, 3137 (1992).

[74] J. K. G. Dhont, An Introduction to Dynamics of Colloids, edited by D. Mobius and R. Miller (Elsevier Science B.V., Amsterdam, 1996).

[75] P. J. Steinhardt, D. R. Nelson, and M. Ronchetti, BondOrientational Order in Liquids and Glasses, Phys. Rev. B 28, 784 (1983).

[76] P. N. Pusey, E. Zaccarelli, C. Valeriani, E. Sanz, W. C. K. Poon, and M. E. Cates, Hard Spheres: Crystallization and Glass Formation, Phil. Trans. R. Soc. A 367, 4993 (2009).

[77] H. Tanaka, Bond Orientational Order in Liquids: Towards a Unified Description of Water-like Anomalies, Liquid-Liquid Transition, Glass Transition, and Crystallization, Eur. Phys. J. E 35, 113 (2012).

[78] See Supplemental Material at http://link.aps.org/ supplemental/10.1103/PhysRevX.11.021056 for video capture of OCULI particles observed under two-channel laser scanning confocal microscopy. Both translational and rotational Brownian motion can be seen. 